\title{
Nonlinear Alfvén waves, discontinuities, proton perpendicular acceleration, and magnetic holes/decreases in interplanetary space and the magnetosphere: intermediate shocks?
}

\author{
B. T. Tsurutani ${ }^{1,2}$, G. S. Lakhina ${ }^{3}$, J. S. Pickett ${ }^{4}$, F. L. Guarnieri ${ }^{2,5}$, N. Lin ${ }^{6}$, and B. E. Goldstein ${ }^{1}$ \\ ${ }^{1}$ Jet Propulsion Laboratory, California Institute of Technology, 4800 Oak Grove Drive, Pasadena, CA, 91109, USA \\ ${ }^{2}$ Space Science Center, University of Southern California, Los Angeles, CA, USA \\ ${ }^{3}$ Indian Institute of Geomagnetism, Mumbai/Bombay, India \\ ${ }^{4}$ Dept. of Physics and Astronomy, University of Iowa, Iowa City, IA, USA \\ ${ }^{5}$ Brazilian National Institute for Space Research (INPE), Av. dos Astronautas, 1.758 - São José dos Campos, SP-12227-010, \\ Brazil \\ ${ }^{6}$ Space Sciences Laboratory,University at Berkeley, Berkeley, CA, USA
}

Received: 10 September 2004 - Revised: 27 January 2005 - Accepted: 28 January 2005 - Published: 18 February 2005

Part of Special Issue "Advances in space environment turbulence"

\begin{abstract}
Alfvén waves, discontinuities, proton perpendicular acceleration and magnetic decreases (MDs) in interplanetary space are shown to be interrelated. Discontinuities are the phase-steepened edges of Alfvén waves. Magnetic decreases are caused by a diamagnetic effect from perpendicularly accelerated (to the magnetic field) protons. The ion acceleration is associated with the dissipation of phasesteepened Alfvén waves, presumably through the Ponderomotive Force. Proton perpendicular heating, through instabilities, lead to the generation of both proton cyclotron waves and mirror mode structures. Electromagnetic and electrostatic electron waves are detected as well. The Alfvén waves are thus found to be both dispersive and dissipative, conditions indicting that they may be intermediate shocks. The resultant "turbulence" created by the Alfvén wave dissipation is quite complex. There are both propagating (waves) and nonpropagating (mirror mode structures and MDs) byproducts. Arguments are presented to indicate that similar processes associated with Alfvén waves are occurring in the magnetosphere. In the magnetosphere, the "turbulence" is even further complicated by the damping of obliquely propagating proton cyclotron waves and the formation of electron holes, a form of solitary waves. Interplanetary Alfvén waves are shown to rapidly phase-steepen at a distance of $1 \mathrm{AU}$ from the Sun. A steepening rate of $\sim 35$ times per wavelength is indicated by Cluster-ACE measurements. Interplanetary (reverse) shock compression of Alfvén waves is noted to cause the rapid formation of MDs on the sunward
\end{abstract}

Correspondence to: B. T. Tsurutani

(bruce.tsurutani@jpl.nasa.gov) side of corotating interaction regions (CIRs). Although much has been learned about the Alfvén wave phase-steepening processfrom space plasma observations, many facets are still not understood. Several of these topics are discussed for the interested researcher. Computer simulations and theoretical developments will be particularly useful in making further progress in this exciting new area.

\section{Introduction}

The microstructure of the interplanetary medium is dominated by the presence of Alfvén waves propagating outward from the sun (Coleman, 1968; Belcher and Davis, 1971; Denskat, et al., 1981; Smith et al., 1995). Although the waves were originally identified over 30 years ago, their properties are not yet fully understood (Medvedev et al., 1997; Goldstein et al., 1999; Hollweg, 1999; Vasquez and Hollweg, 1999, 2001; Buti et al., 1999, 2001). Close to the sun, the Alfvén wave phase speed is extremely high $\left(\sim 1200 \mathrm{~km} / \mathrm{s}\right.$ at $\left.4 \mathrm{R}_{s}\right)$ compared with that at $1 \mathrm{AU}(\sim 50$ $70 \mathrm{~km} / \mathrm{s}$ ). Far from the sun, the waves are convected by the solar wind which has speeds varying from $\sim 400 \mathrm{~km} / \mathrm{s}$ (the slow solar wind) to $\sim 750 \mathrm{~km} / \mathrm{s}$ (the fast solar wind emanating from coronal holes; Phillips et al., 1996; McComas et al., 2002). To conserve energy flux density (assuming no dissipation), the wave amplitude becomes much greater with outward convection/propagation. Thus, the expanding solar wind is the driver for the waves becoming nonlinear. Interplanetary Alfvén waves have been shown to be dispersive 
and compressive (Medvedev and Diamond, 1996; Medvedev et al., 1997; Vasquez and Hollweg, 2001; Buti et al., 2001). Theoretical models such as Medvedev and Diamond (1996) and Medvedev et al. (1997) have indicated that mode coupling between Alfvén waves and ion acoustic waves would result in heating of the local solar wind plasma via damping of the ion acoustic waves. The magnitude of this coupling and therefore the rate of energy transport from Alfvén wave energy to plasma thermal energy is presently unclear however.

The interplanetary magnetic field (IMF) varies greatly on different temporal and spatial scales as indicted by several heliospheric spacecraft (Helios 1 and 2, Pioneer 10 and 11, Voyagers 1 and 2, Mariner 10, Ulysses, etc.). One of the fundamental microstructures in the IMF are directional discontinuities (DDs) where the magnetic field direction changes sharply on time scales of seconds (at 1 AU). Burlaga (1969) defined DDs as the changes in magnetic field direction of $>30^{\circ}$ in $\leq 30 \mathrm{~s}$. Two basic types of DDs, namely tangential (TDs) and rotational (RDs) (see Landau and Lifschitz, 1960; Colburn and Sonett, 1966), have been observed in interplanetary space (Belcher and Davis, 1971; Smith, 1973a, b; Belcher and Solodyna, 1975; Burlaga et al., 1977; Tsurutani et al., 1979a, b; Neubauer and Barnstorf, 1981; Neugebauer et al., 1984). Rotational discontinuities are sharp changes in the magnetic field direction, essentially propagating "kinks" in the magnetic field, and for many cases, are believed to be phase-steepened edges of Alfvén waves (Tsurutani et al., 1994, 1996). On the other hand, TDs are nonpropagating structures in the solar wind which separate totally different plasma regions and, as such, could be potential sites of magnetic reconnection as well as other plasma instabilities (Tsurutani and Smith, 1979a; Lepping and Behannon, 1986; Ho et al., 1995).

Ulysses observations (Tsurutani et al., 1996) have also indicated that there are 5 to 10 times greater number of directional discontinuities in coronal hole high speed streams than in the slow solar wind. A plausible explanation is that Alfvén waves are often phase-steepened, e.g. one edge of an Alfvén wave has a rotational discontinuity which is part of the wave. Phase-steepening of nonlinear cometary plasma waves have also been reported in the literature (Tsurutani et al., 1987, 1990, 1995, 1997a, b; Glassmeier and Neubauer, 1993; Neubauer et al., 1993; Mazelle et al., 1995). This has been noted for both magnetosonic (right-hand) waves as well as for left-hand (ion cyclotron) waves. Wave steepening is an alternative process to wave-wave interactions or MHD wave cascading for the generation of the turbulent spectra that are measured in the interplanetary medium (Tu and Marsch, 1995; Goldstein and Roberts, 1995; Matthaeus et al., 1995; Bhattacharjee et al., 1998; 1999; Horbury and Balogh, 2001).

Small scale depressions in the interplanetary magnetic field have been called magnetic holes (MHs) and magnetic decreases (MDs) (Turner et al., 1977; Winterhalter et al., 1994, 2000; Fränz et al., 2000; Tsurutani and Ho, 1999; Neugebauer et al., 2001). The MHs/MDs are pressure balance structures, i.e. the magnetic field plus plasma total pres- sure remains constant across the structures, to first order. Similar structures have been detected within the Earth's magnetospheric field but have been given different names (magnetic bubbles, etc: see Tsurutani et al., 2003 for references). It has recently been shown that MHs/MDs are located at the phase-steepened edges of nonlinear Alfvén waves (Tsurutani et al., 2002a), and it has been speculated that perpendicular plasma heating by the ponderomotive force associated with the phase-steepened wave edges cause the formation of MHs/MDs (Tsurutani et al., 2002b). It is found that the perpendicular (to the ambient magnetic field) temperatures are $\sim 20 \%$ higher inside the MHs/MDs, but the parallel temperatures are essentially the same (see also Fränz et al., 2000; Neugebauer et al., 2001). Thus with only slight temperature increases and often large magnetic field magnitude decreases, the densities are substantially higher within MHs/MDs (Winterhalter et al., 1994a). Further, the differential $\mathrm{He}^{++}$-proton streaming, which is present in the regular fast solar wind, disappears within MDs (Neugebauer et al., 2001). At this time, the explanation for this feature is not understood. Better understanding of MD formation and evolution, induced micro-instabilities and wave-particle interactions will help clarify this process.

Since MHs/MDs are high density interplanetary structures being convected by the solar wind, their impact on the Earth's magnetosphere will be manifested as significant ram pressure increases. At this time, it is unclear how large MHs/MDs are in spatial scale. If they are smaller than the size of the magnetosphere, the magnetospheric compression will be asymmetric. This should be detectable by using satellite UV imaging data (see Zhou and Tsurutani, 1999 for examples of shock-compression auroras). Multipoint Cluster measurements with ACE measurements will provide information on the size of MHs/MDs for the first time.

Several mechanisms have been proposed to create MHs/MDs. Winterhalter et al. (1994a) proposed the mirror instability generates "linear" magnetic holes. Linear magnetic holes are those in which the magnetic field changes direction by $10^{\circ}$ or less. Baumgärtel et al. (1999) have proposed a generation mechanism in terms of Alfvén solitons. Vasquez and Hollweg (1999) have proposed wavewave interactions for the creation of MHs/MDs. Neugebauer et al. (2001) have suggested that a variety of beam microinstabilities that give rise to proton perpendicular heating, leading to the generation of these structures. Medvedev et al. (1997) and Buti et al. (2001) have proposed that evolution of nonlinear Alfvén waves create compressional features. Tsurutani et al. (2002b) have suggested that it is the Ponderomotive Force associated with the high frequency components of the Alfvén wave phase steepened edges that is the mechanism for proton perpendicular heating. However none of these mechanisms explain both the higher proton densities and proton perpendicular temperatures that are present within the MHs/MDs (Winterhalter et al., 1994a), thus the evolution of MHs/MDs remain to be explained.

The time cadence of the Ulysses plasma data taken is extremely slow $(4 \mathrm{~min})$. To analyze plasma data within 


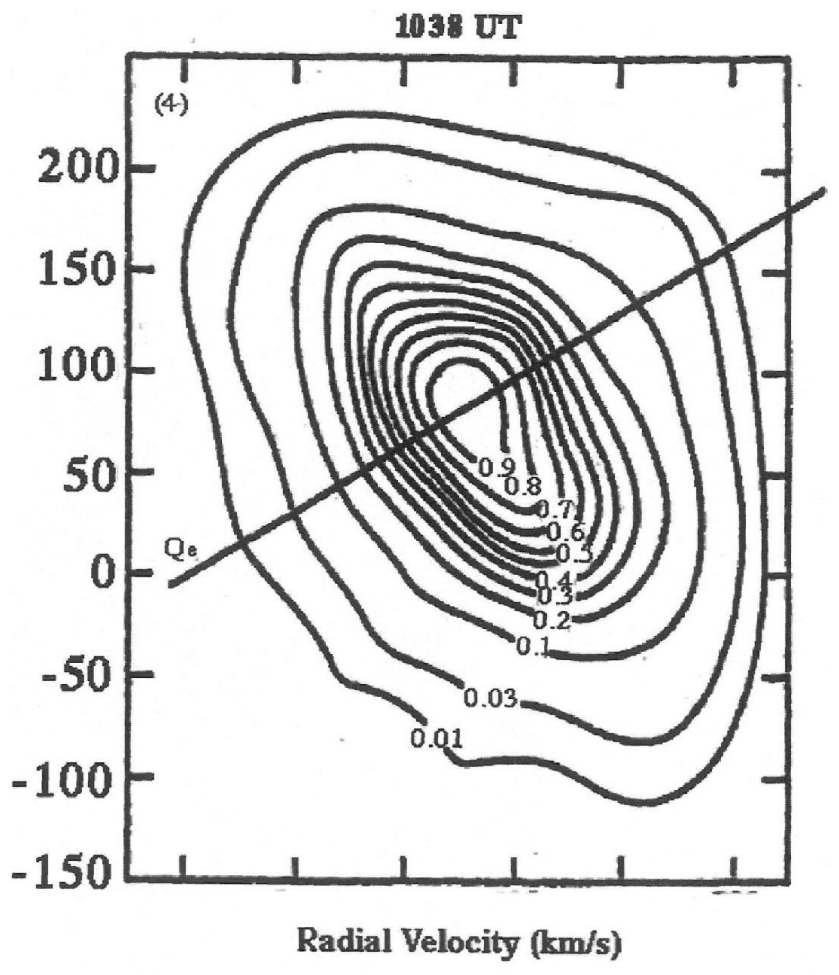

From Bame et a1., GRL, Sep. 1975

Fig. 1. A proton two-dimensional distribution function in the solar wind. The ambient magnetic field is indicated by the straight line. Taken from Bame et al. (1975).

MHs/MDs, large scale MDs were analyzed. In contrast, Cluster plasma data has a $2 \mathrm{~s}$ cadence. Thus in the future, analysis of the Cluster plasma data should be used to determine how the protons, He++ (and possibly electrons) vary from $\mathrm{MH} / \mathrm{MD}$ edge to edge, and what relationship heating plays relative to the phase steepened edges of the MHs/MDs.

\section{Results}

2.1 Historical perspective of some interplanetary plasma features

Several interplanetary plasma phenomena have been reported in the 1960s and 1970s, at the beginning of the space era. A short review of phenomena pertinent to this paper will be given below.

Figure 1 shows a proton two-dimensional distribution function taken from Bame et al. (1975). This measurement was taken by a VELA satellite in interplanetary space at $1 \mathrm{AU}$. The direction of the interplanetary magnetic field is indicated by the straight line drawn through the isovelocity curves. The figure shows that the distribution is broader in the direction perpendicular to $\boldsymbol{B}$ compared to that parallel to $\boldsymbol{B}$, indicating that the perpendicular "temperature" is higher than the parallel "temperature". Bame et al. (1975) concluded that local (perpendicular) plasma heating was oc-

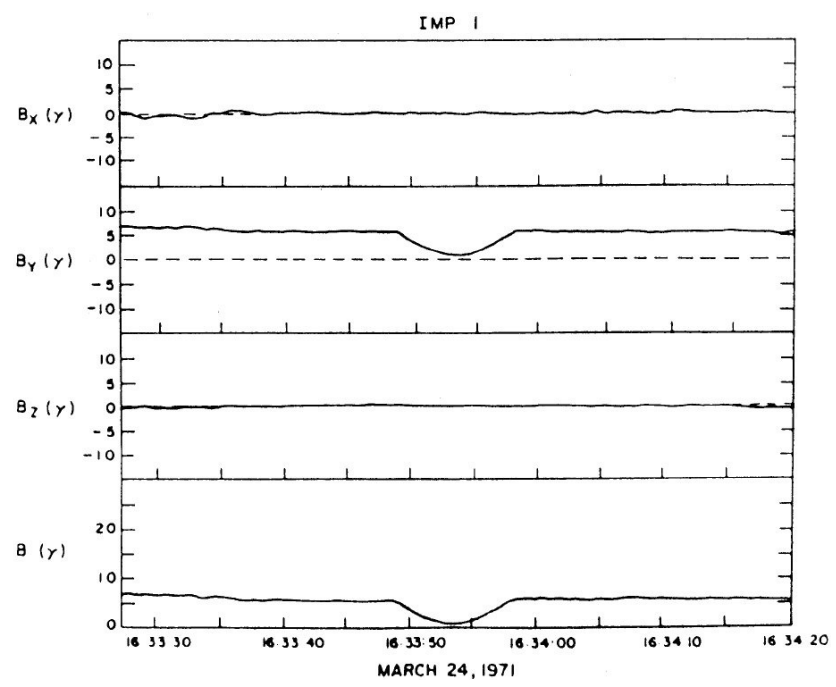

Fig. 2. An example of a magnetic hole $(\mathrm{MH})$ at $1 \mathrm{AU}$. Taken from Turner et al. (1977).

curring in the solar wind at 1 AU. Later Marsch et al. (1982) demonstrated that such heating was occurring in high speed solar wind streams throughout the inner heliosphere, from 0.3 to $1.0 \mathrm{AU}$ (Helios 1 and 2 observations).

Figure 2 shows a "magnetic hole" $(\mathrm{MH})$ detected in interplanetary space at $1 \mathrm{AU}$, reported by Turner et al. (1977). The coordinate system is GSE. Magnetic holes are identified by field magnitude decreases, such as the one centered at $\sim 16: 33: 54$ UT on 4 March 1971. This particular event was only $\sim 10 \mathrm{~s}$ wide, but much larger events have been noted in other works (magnetic decreases will be discussed later). Winterhalter et al. (1994; 1995), using Ulysses plasma and field data have shown that plasma thermal pressure makes up for the missing magnetic pressure within the hole, thus MHs are pressure equilibrium structures. Winterhalter et al. (2000) have called magnetic holes with little or no field directional changes "linear holes".

It should be noted that similar field decrease structures were detected in the magnetosphere, but were given different names: "holes" (Sugiura et al., 1969; Treumann et al.,1990), "dropouts" (Russell and Elphic, 1978), "magnetic cavities" (Luhr and Klocker, 1987), and "magnetic bubbles" (Stasiewicz et al., 2001).

Figure 3 are examples of somewhat larger structures called magnetic decreases (MDs) detected in the Ulysses data at a heliocentric distance of $\sim 2.0 \mathrm{AU}$ from the sun. The coordinate system is the RTN heliospheric system. R points radially from the sun to the spacecraft, $T=\Omega \times R /|\Omega \times R|$, where $\Omega$ is the spin axis of the sun, and $\mathrm{N}$ completes the right hand system. These structures were originally called MDs because of the often-present sharp decreases at the two boundaries of the structures. See the examples in the top and bottom panels of the figure (however, Tsurutani et al., 2002a, using statistical arguments, have argued that MHs and MDs are most probably the same phenomena). These MD events have a variety 

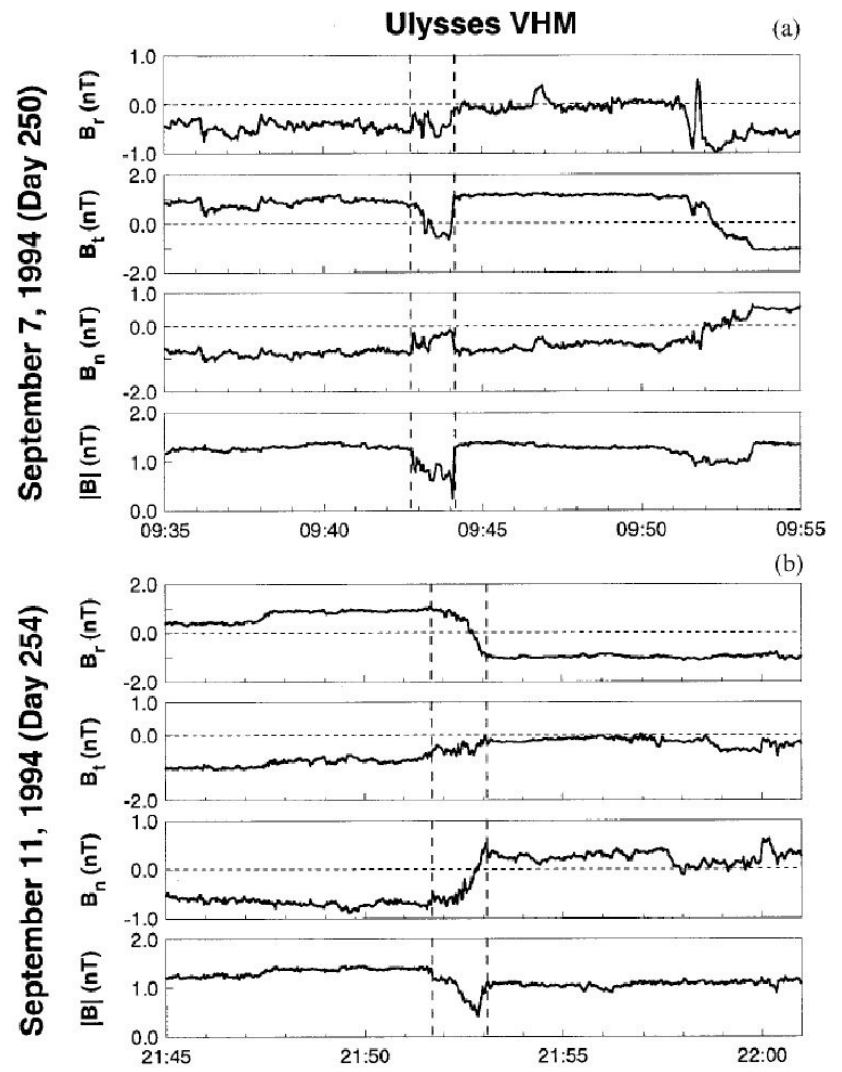

(c)

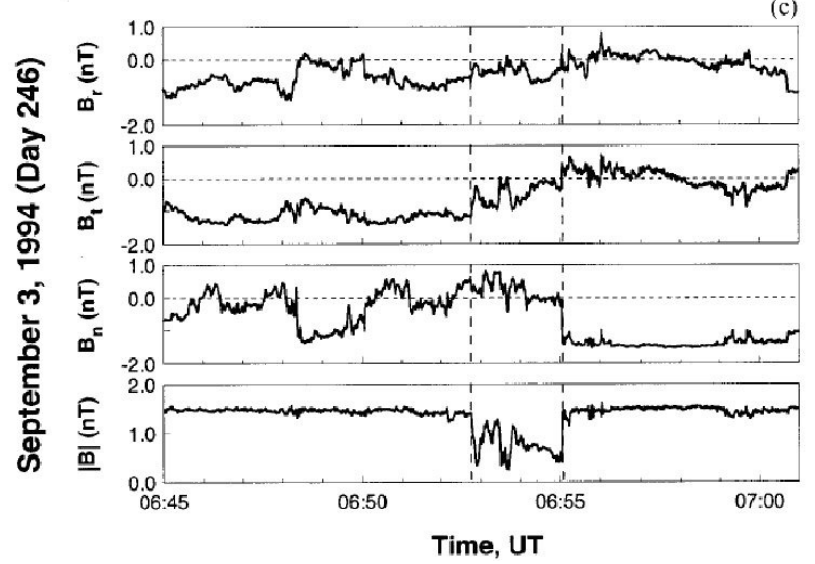

Fig. 3. Examples of magnetic decreases (MDs) at $\sim 2$ AU. Note the sharp field decreases at the edges of the MDs in the top and bottom panels. Taken from Tsurutani and Ho, 1999.

of magnetic field variations across their structure. The top example has almost no field directional change nor magnitude change from one side of the MD to the other (a "linear" MD), similar to the MH shown in Fig. 2. The middle case in Fig. 3 has both a magnetic field directional change and a magnitude change across the structure. The bottom example has a field change in primarily one axis, that of $B_{n}$.

The nature of the sharp decreases bounding the MDs is presently not well understood. Farrugia et al. (2001) have examined one sharp boundary of an extended MD and have suggested that the event was a slow shock. Whether these sharp boundaries are more prevalent at larger distances from the sun or not is not known at this time.

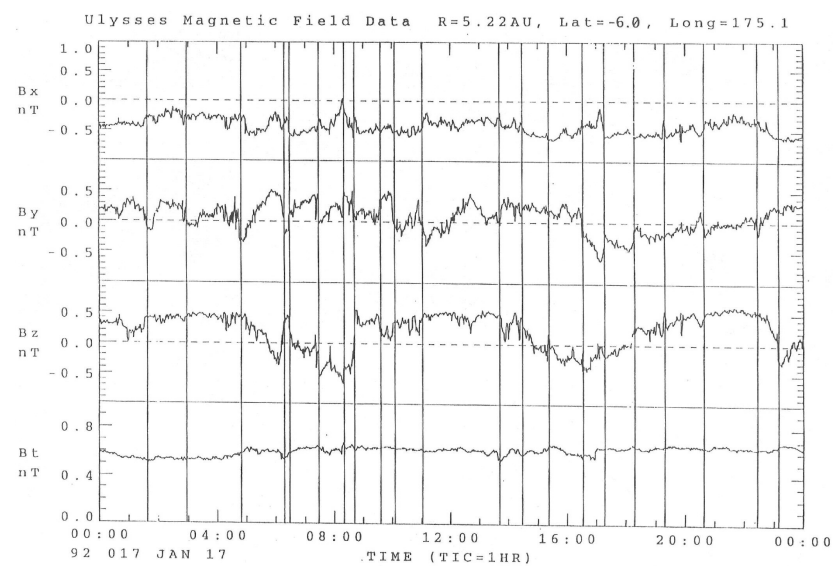

Fig. 4. Interplanetary directional discontinuities (vertical lines).

Figure 4 shows interplanetary "directional" discontinuities (DDs) in the Ulysses magnetometer data set. The discontinuities are identified by vertical lines. One of the first reviews on this topic was by Colburn and Sonett (1966). Directional discontinuities are sharp directional changes in the magnetic field (typically $\Delta \boldsymbol{B}>0.5|B|$ is used as a criterion) and occur frequently in interplanetary space. At $1 \mathrm{AU}$, they occur at a rate of 1 or 2 per hour. DDs and Alfvén waves are conspicuously absent within magnetic clouds, specific episodic solar ejecta. This property has been used for the identification of magnetic clouds.

Ever since directional discontinuities were first discovered (Ness, et al., 1966; Colburn and Sonett, 1966; Siscoe et al., 1968), there has been a lively debate over whether they are rotational or tangential (or both) in nature. Some arguments can be found in Neugebauer et al. (1984). Smith (1973a, b) devised a method to distinguish between the two types based on determining the normal to the structures. The latter was done using the minimum variance method (Sonnerup and Cahill, 1967). Smith (1973b) sorted discontinuities into a two-dimensional phase space. Discontinuities with large magnetic field components along the normal direction have rotational discontinuity properties (a sharply crested Alfvén wave). Those with small or no field components along the normal are consistent with tangential discontinuities (TDs). A TD is a structure separating two dissimilar sets of plasmas and fields. Ideally, there is no plasma flow (or field) crossing the structure. See discussions in Horbury et al. (2001) and Knetter et al. (2004).

In Fig. 4, it can be noted that there is little or no field magnitude changes across these directional discontinuities. However, it will later be shown that this is not always the case, especially at larger distances from the sun.

Coleman (1968) and Belcher and Davis (1971) first discovered the existence of Alfvén waves in interplanetary space. This was clearly demonstrated by showing the strong correlation between variations in the components of the magnetic field and components of the solar wind velocity vector. By identifying the sign of the cross correlation coefficients, 


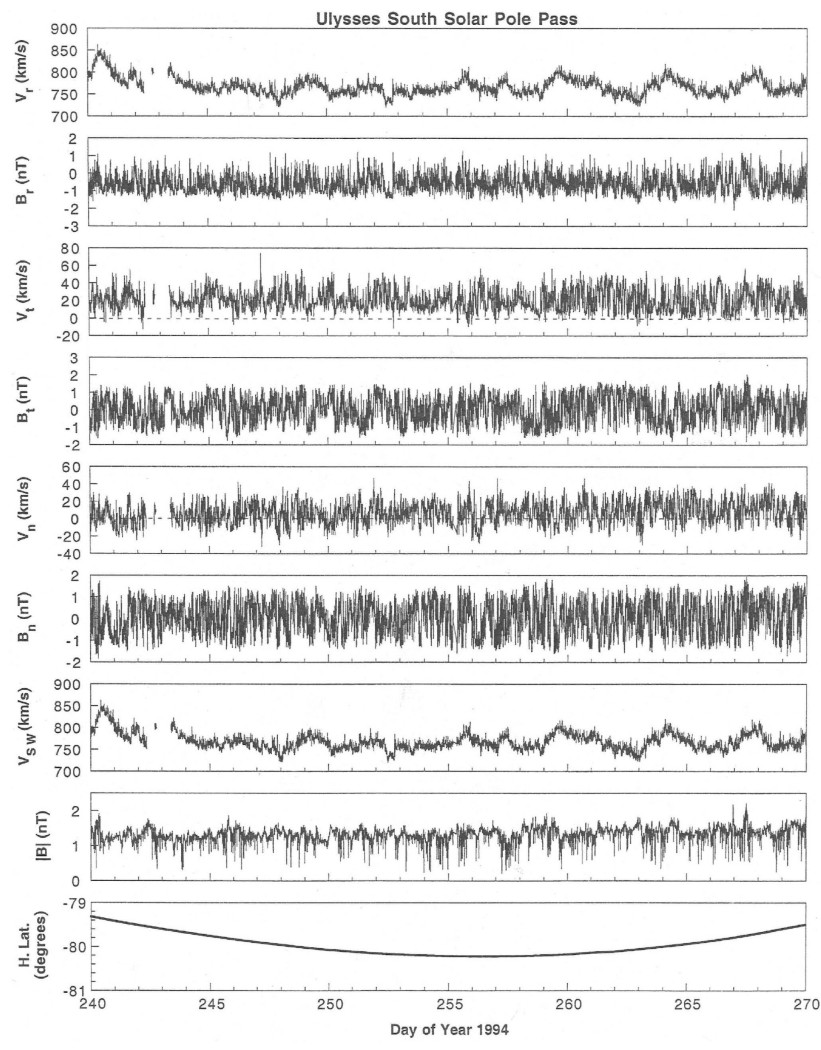

Fig. 5. The magnetic field and solar wind velocity during the first Ulysses polar pass. The large amplitude fluctuations in $B$ and $V$ are Alfvén waves propagating outward from the sun. The decreases in the field magnitude are the MDs shown in Fig. 3.

Belcher and Davis (1971) were able to show that the waves were propagating outward from the sun.

Alfvén waves are noted to have particularly large amplitudes in high speed solar wind streams. Figure 5 shows an example of a 30-day Ulysses interval taken over the southern solar pole. The magnitude of the magnetic fluctuations are $\Delta \boldsymbol{B} /|B| \sim 1$ to 2 . In this particular case, Ulysses was immersed in a high speed stream emanating from a large polar coronal hole. The speed of the solar wind was nearly constant, with a value of 750 to $800 \mathrm{~km} \mathrm{~s}^{-1}$ (third from the bottom panel).

Sharp decreases in the magnetic field magnitude can be noted in the next to bottom panel of the figure. On this scale, these appear as downward pointing (decreasing) spikes. There are increasing spikes, but these are much smaller in magnitude. There are also broader, small amplitude magnetic field magnitude increases present (this will be discussed later).

The rate of directional discontinuity occurrence was shown to be well correlated with the solar wind speed (not shown to save space). It was found that when Ulysses was in a high speed stream, the occurrence rate of DDs was 5 to 10 times higher than when Ulysses was in a slow speed stream. The DD thickness distribution was used to determine whether solar wind convection alone could explain the
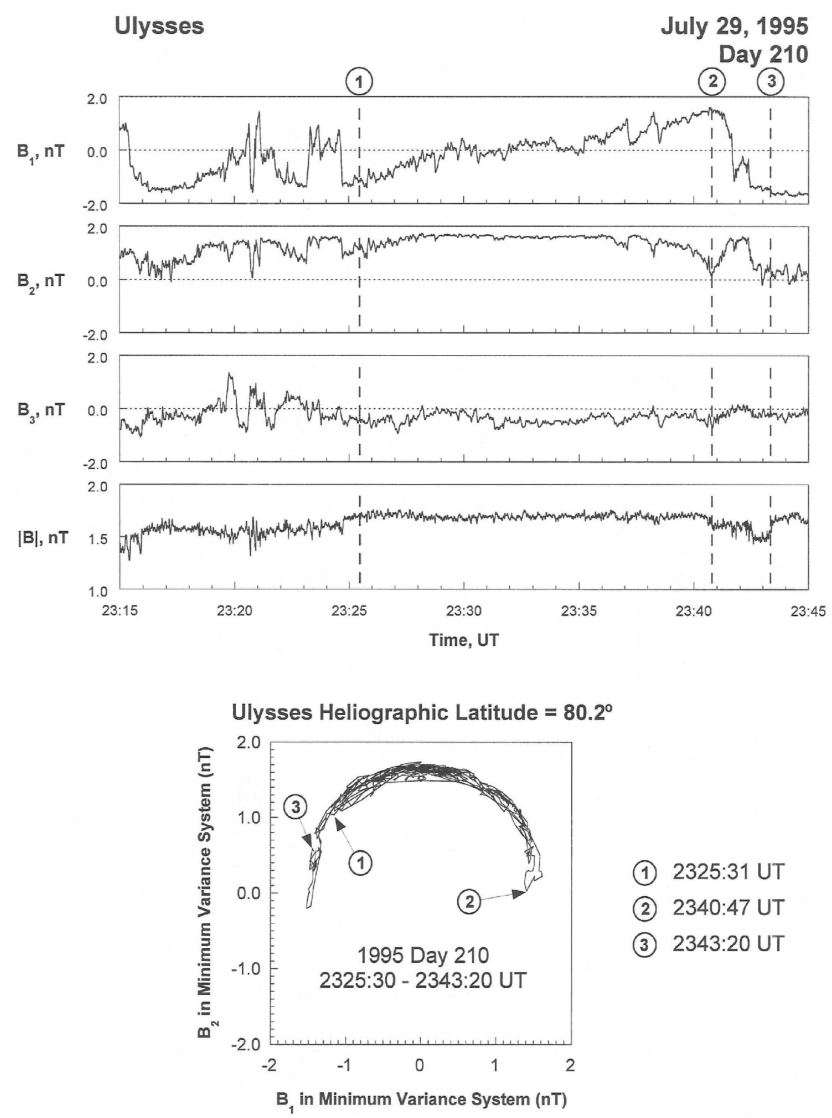

Fig. 6. The relationship between Alfvén waves and directional discontinuities. Taken from Tsurutani et al. (1994).

increase. It was determined that convection could explain a factor of $\sim 2$ increase, but not the full 5 to 10 times increase (Tsurutani et al., 1996).

Figure 6 shows a relationship between one cycle of an Alfvén wave and DDs. The top panel is the magnetic field given in minimum variance coordinates. Smith and Tsurutani (1976) first showed that the minimum variance method could be applied to electromagnetic waves to determine the wave direction of propagation and the wave polarization. The bottom panel is a hodogram of the wave corresponding to the interval between points 1 and 3 . In the top panel, the magnetic field rotates slowly and smoothly between points 1 and 2. This is noted in the $B_{1}$ component, the field component in the maximum variance direction. Between points 2 and 3 there are two sharp rotations (DDs).

The hodogram shows the field $B_{1}-B_{2}$ variation ( $B_{2}$ is the field component along the intermediate variance direction). From point 1 to 2 , the field rotates in an arc, and from point 2 to 3 , the field rotates back to the original position, tracing back along the same arc. The overall rotation from point 1 to 3 completes $360^{\circ}$ rotation in phase. The DDs comprise the phase-steepened edge of the Alfvén wave (here there are two distinct DDs at the edge of the Alfvén wave. Typically there is only one). The Alfvén wave is thus "arc-polarized". This corresponds to a large amplitude rotation of the magnetic 

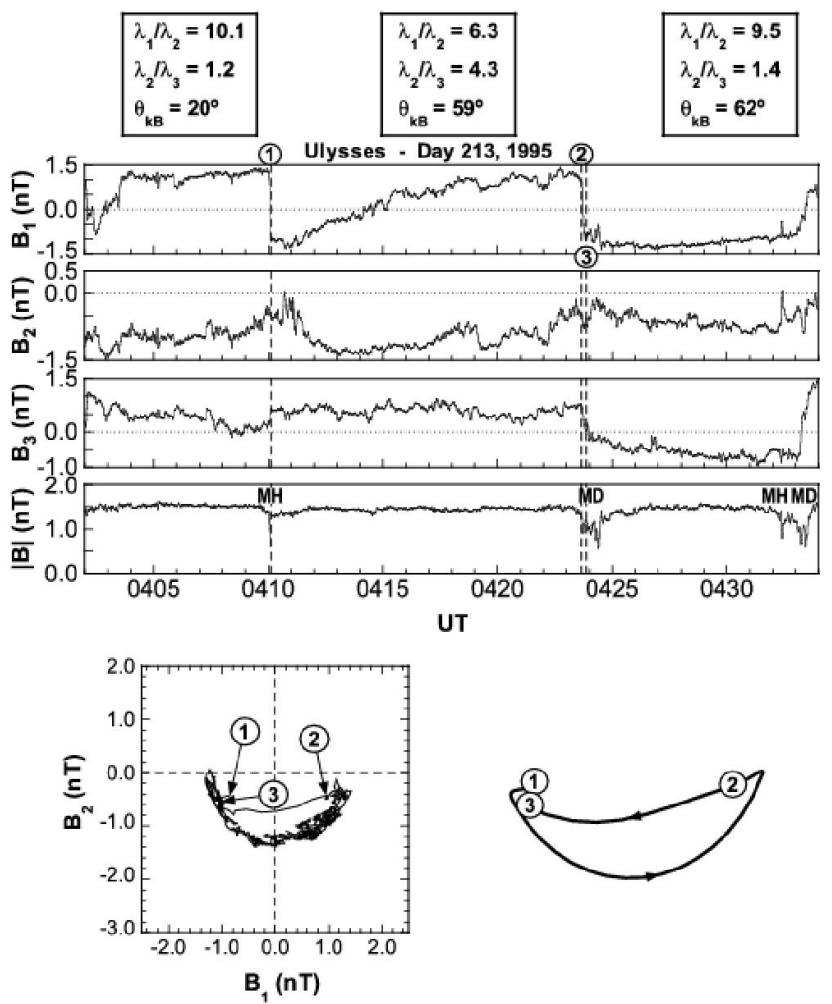

Fig. 7. The relationship between phase-steepened Alfvén waves and MHs and MDs. The MHs/MDs are detected at the phase-steepened edges of the waves. Taken from Tsurutani et al. (2002a).

field vector, keeping the field magnitude constant, to first order (imagine a field vector centered at the origin, with the tip of the vector rotating on the surface of a sphere). Arcpolarization may be the large amplitude extension of small amplitude transverse linearly polarized waves.

For a reader who would like to see examples of phasesteepened waves that are easier to visualize, we refer them to the cometary wave cases. Both phase-steepened circularly polarized right-hand (magnetosonic) waves and left-hand circularly polarized waves have been studied (Tsurutani et al., 1997a, b). Phase-steepened waves have also been detected in planetary foreshocks (Fairfield, 1969; Russell et al., 1971; Hoppe et al., 1981).

The magnetic field magnitude in Fig. 6 is not exactly constant. There is a small $\sim 10 \%$ decrease in the interval between points 2 and 3. Also, the field magnitude is slightly elevated between points 1 and 2 from the region that occurs prior to point 1 . We will come back to these points later.

The relationship between phase-steepened Alfvén waves and MDs and MHs is shown in Fig. 7. The top panel shows three cycles of a phase-steepened Alfvén wave. The properties of the individual wave cycles are given in the inserts at the top (over each cycle). The MDs/MHs are indicated above the field magnitude plot. MDs/MHs are noted to be associated with the phase-steepened edges (DDs) of the Alfvén waves for all three wave cycles.

\section{Ulysses - Days 208-216, 1995}
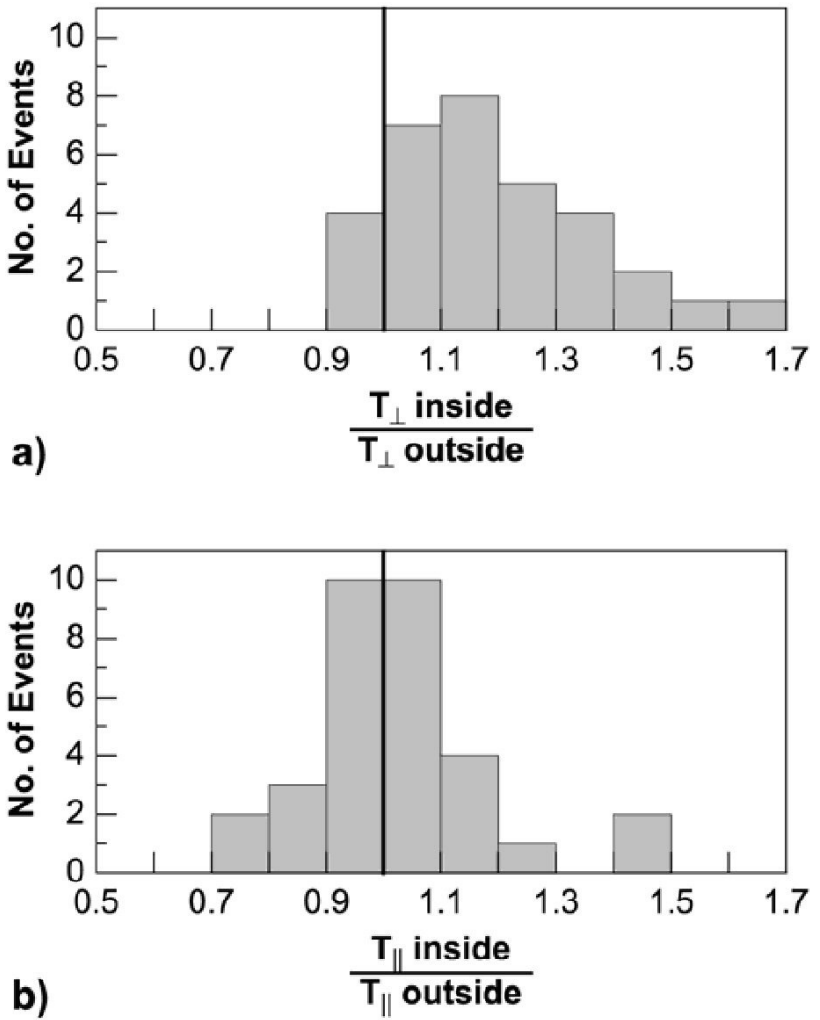

Fig. 8. The ratios of the inside to outside (the MD) parallel and perpendicular proton temperatures. The proton perpendicular temperatures are higher inside the MD than outside the structure.

The proton distribution functions were examined for a number of temporally broad MDs. This was done (rather than to examine the much shorter MHs) because the cadence of the plasma detector onboard Ulysses is much slower than the magnetometer. Assuming that the plasma has a biMaxwellian form, temperatures parallel to $\left(T_{\|}\right)$and perpendicular to $\left(T_{\perp}\right)$ the magnetic field were calculated. For comparative purposes, a point near but outside the MD was selected to measure $\left(T_{\|}\right)$and $\left(T_{\perp}\right)$. These "temperature" values were used to determine the differences inside the MD from those of the ambient (outside the MD).

The results of the analyses are given in Fig. 8. The bottom panel gives the ratio of the parallel temperature inside the MD to the parallel temperature outside the MD. The ratio (for each MD) has been binned and is plotted in histogram format. The center of the distribution is close to 1.0. Thus within statistical variability, it appears as if the parallel temperatures inside and outside the MD are about the same.

The top panel shows the results for the proton perpendicular temperatures, analyzed in the same manner. It can be noted that the perpendicular temperatures inside the MDs are generally higher than that outside. One implication is that proton perpendicular heating is occurring, and the process is associated with the phase-steepened edges of the Alfvén waves. 


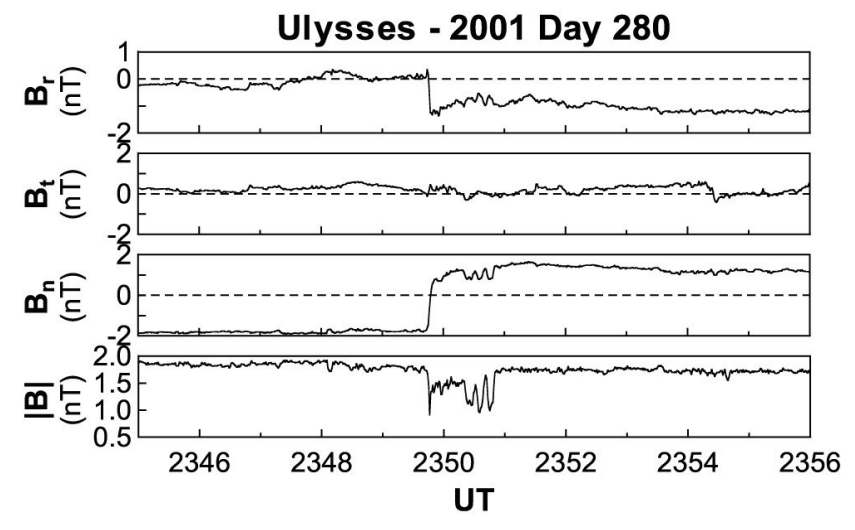

Fig. 9. Mirror mode structures within a MD. Several oscillations can be noted from 23:50:20 to 23:50:50 UT.

It should be noted that the $T_{\perp} / T_{\|}$ratio is not always $>1.0$ inside MDs. This occurs only $\sim 20 \%$ of the time. This may be due to adiabatic decompression effects that also must be taken into account.

Taken from the observation of plasma waves/structures within MDs/MHs, a number of plasma instabilities have been inferred to occur. Tsurutani et al. (2002b) have shown two types of waves/structures that would be associated with proton $T_{\perp} / T_{\|}>1$ distributions: electromagnetic proton cyclotron waves and mirror mode structures. An example of a few cycles of a mirror mode structure is shown in Fig. 9 at $\sim 23: 50: 40$ UT. It should be noted that while proton cyclotron waves are propagating waves, mirror mode structures are not, in the conventional sense. Their $\boldsymbol{k}$ values are usually found to be highly oblique to the ambient magnetic field, indicating that they are primarily convected structures. The MDs and MHs themselves are thought to be generated by the diamagnetic effect of the perpendicularly heated protons. As such, these large-scale diamagnetic cavities would also be convected by the solar wind and not be a propagating phenomenon.

Figure 10 shows the electric components of ELF/VLF plasma waves (in red) associated with MHs/MDs. This is an interval when Ulysses was in a high speed stream over the south solar pole. Various wave frequency channels from $3.5 \mathrm{~Hz}$ to $8.2 \mathrm{kHz}$ are shown. The magnetic field magnitude is indicated in green and is repeated for each different wave frequency. The computer-identified directional discontinuities (Tsurutani and Smith, 1979) are indicated by the vertical lines. The strong relationship between the occurrence of directional discontinuities and $\mathrm{MHs} / \mathrm{MDs}$ can be noted. Broader MD intervals are also found in the $|B|$ plots. Wave enhancements are noted to occur at MHs and within the broader MDs.

Specific electron wave modes have been identified through the determination of the wave frequency relative to electron cyclotron and the electron plasma frequencies and also the direction of maximum wave $|E|$ amplitude relative to the ambient magnetic field direction. Two modes have been identified: electron whistler mode and longitudinal Langmuir

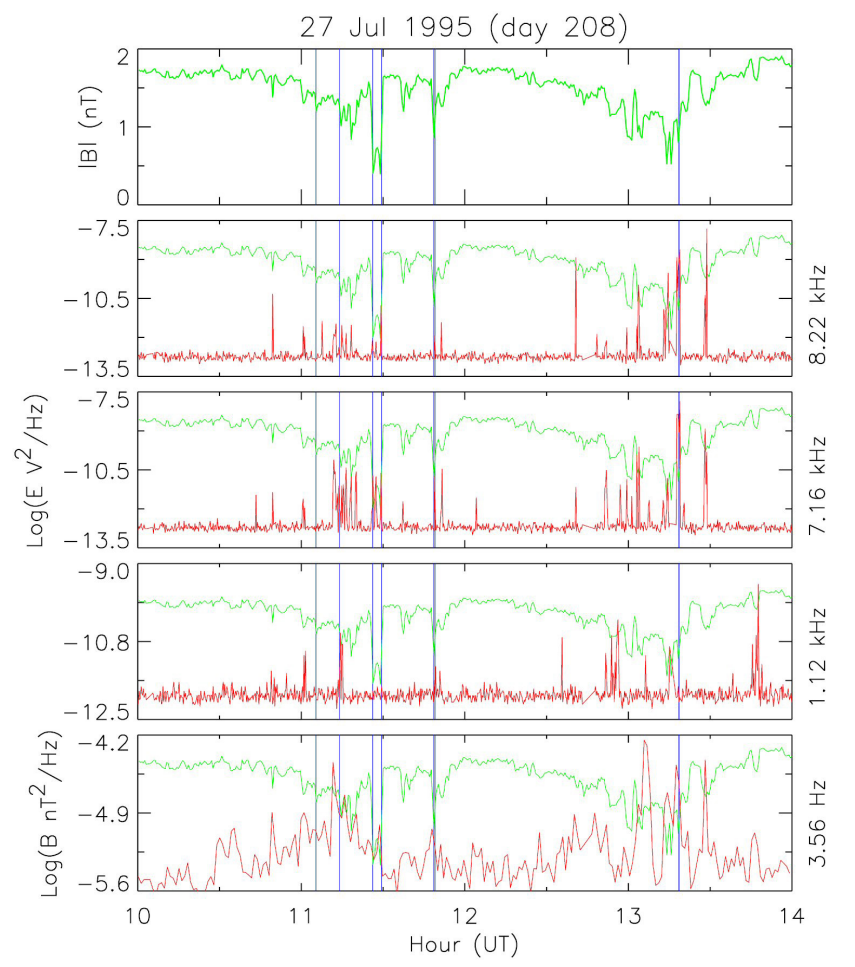

Fig. 10. The relationship between electrostatic noise between 1.12 and $8.22 \mathrm{kHz}$ and $\mathrm{MHs} / \mathrm{MDs}$.

waves. The standard sources of free energy for these two modes are the $T_{e \perp} / T_{e \|}>1$ electron temperature anisotropy instability and an electron beam instability, respectively. Similar results were obtained from other MH intervals (Lin et al., 1995; 1996; MacDowall et al., 1996).

The proton perpendicular velocity distribution is shown in Fig. 11 for one MD event. The red curve is the distribution just outside the MD. The blue curve is the distribution within the MD. It can be noted that neither distribution is Maxwellian (thus in the previous sections where we discussed proton "temperatures", this was clearly a simplification). This is typical for all cases examined. What is of interest is the blue curve relative to the red curve. The blue curve at speeds above $40 \mathrm{~km} / \mathrm{s}$ are everywhere higher than the red curve. This indicates that the entire proton distribution is accelerated and not just one small interval of velocity space (as happens for resonant interactions). See also comments by Neugebauer et al. (2001).

A mechanism for plasma heating at the phase-steepened edges of Alfvén waves is the Ponderomotive Force (Landau and Lifschitz, 1960; Lee and Parks, 1983; Li and Temerin, 1993). Tsurutani et al. (2002b) and Dasgupta et al. (2003) have shown that for one specific wave case, the ambipolar acceleration for protons should be greater in the perpendicular to $\boldsymbol{B}_{0}$ direction than the parallel to $\boldsymbol{B}_{0}$ direction by a factor of $\sim 3$. Clearly for other wave cases, parallel heating can dominate as well.

The occurrence of MHs is not always uniform, but is higher in intervals of corotating fast streams (Winterhal- 


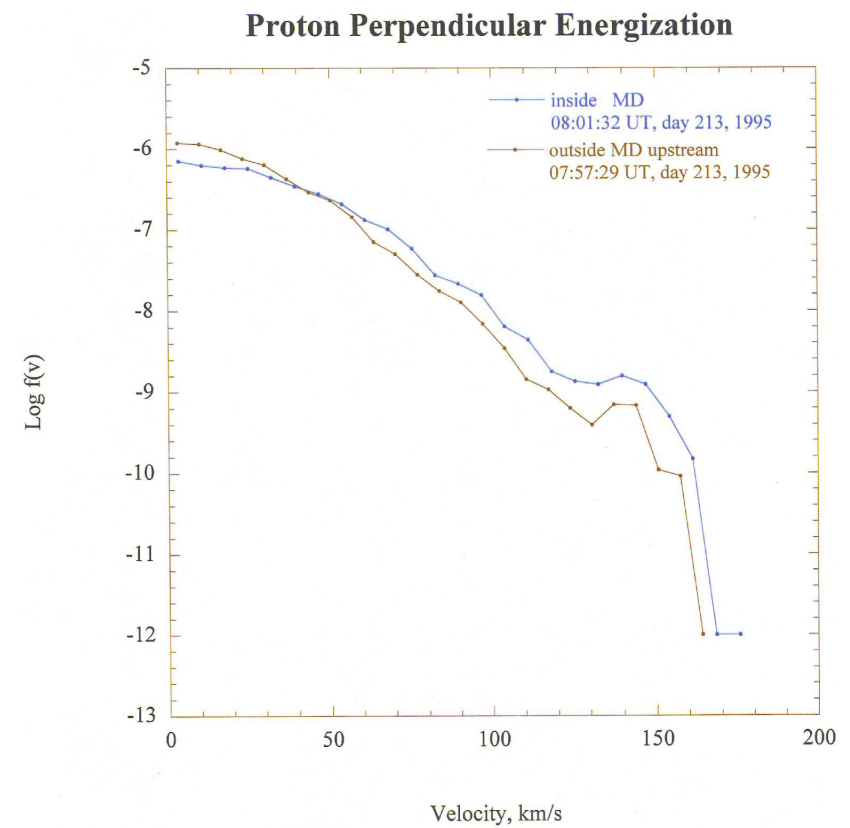

Fig. 11. The proton velocity distributions inside the MD and just outside the MD. The proton velocities inside the MD are enhanced at speeds everywhere above $40 \mathrm{~km} / \mathrm{s}$.

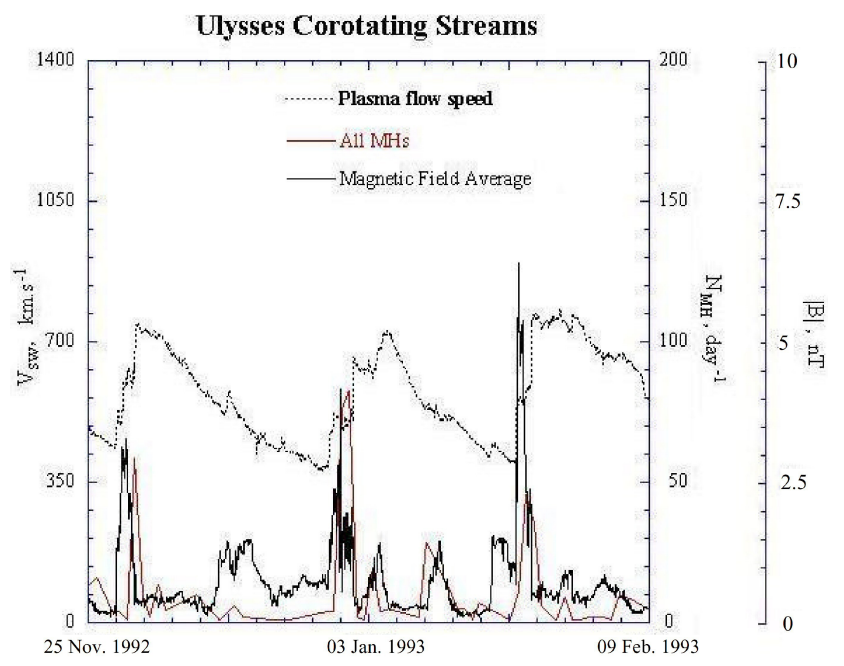

Fig. 12. The solar wind speed, magnetic field intensity and MH occurrence rate for Ulysses at midlatitudes. There are three major Corotating Interaction Regions (CIRs) which are found at the leading edges of the high speed streams. The abrupt field increases and decreases bounding the CIRs are forward and reverse (fast) shocks. The MH occurrence rate has maxima at each of the three CIRs. The peaks in the maxima are found in the trailing portions of the CIRs, close to the reverse shocks.

ter et al., 2000). More specifically, the highest occurrence rates of $\mathrm{MHs}$ are found in Corotating Interaction Regions (CIRs). Figure 12 shows the interrelationship between the fast solar wind streams, the CIRs formed by the fast streamslow stream interactions and the MH occurrence rate. Three high-speed streams and three CIRs are shown in the figure.

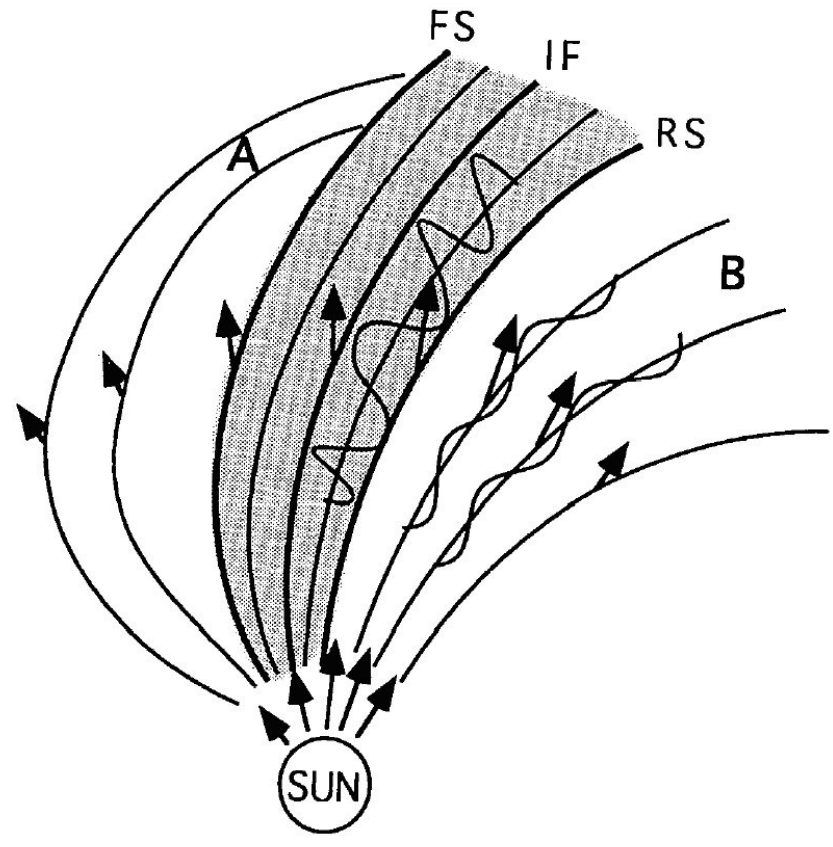

Fig. 13. A schematic of a CIR.

The high magnetic field magnitudes defining the CIRs are noted at the leading antisunward (left-hand edge) of the fast streams. The field increase and subsequent decrease of each CIR (boxcar shape) are time-coincident with two increases in the solar wind speed. This can be noted on the solar wind stream leading (antisunward) edge. The increase in the field of the CIR corresponds to a (fast) forward shock propagating away from the sun. The subsequent decrease in the field is a (fast) reverse shock propagating backwards towards the sun. Because the solar wind (convection) speed is much greater than the reverse shock speed, the reverse shock is convected outward past Ulysses. The original work on CIR formation and its shocks can be found in Smith and Wolfe (1976). More recent discussion can be found in Pizzo (1985) and Balogh et al. (2002).

An interesting feature of the figure is that the MHs are not uniformly distributed throughout the CIRs, but they occur more frequently on the sunward part of the CIR. This location is closer to the reverse shock rather than the forward shock.

A schematic of a CIR will be useful to understand the implications of such an offset. In Fig. 13, region A corresponds to the slow solar wind and region B the fast solar wind. The CIR, the compressed field region, is indicated by shading. The forward shock, interface (tangential?) discontinuity, and the reverse shock are denoted by the designations FS, IF and RS, respectively. The plasma and field between the FS and the IF correspond to the shocked (compressed) and accelerated slow solar wind. The more important region is the volume of material between the IF and the RS. This corresponds to the (reverse) shocked and decelerated fast solar wind plasma. The schematic indicates that Alfvén waves which are present in the fast solar wind will be amplified by 


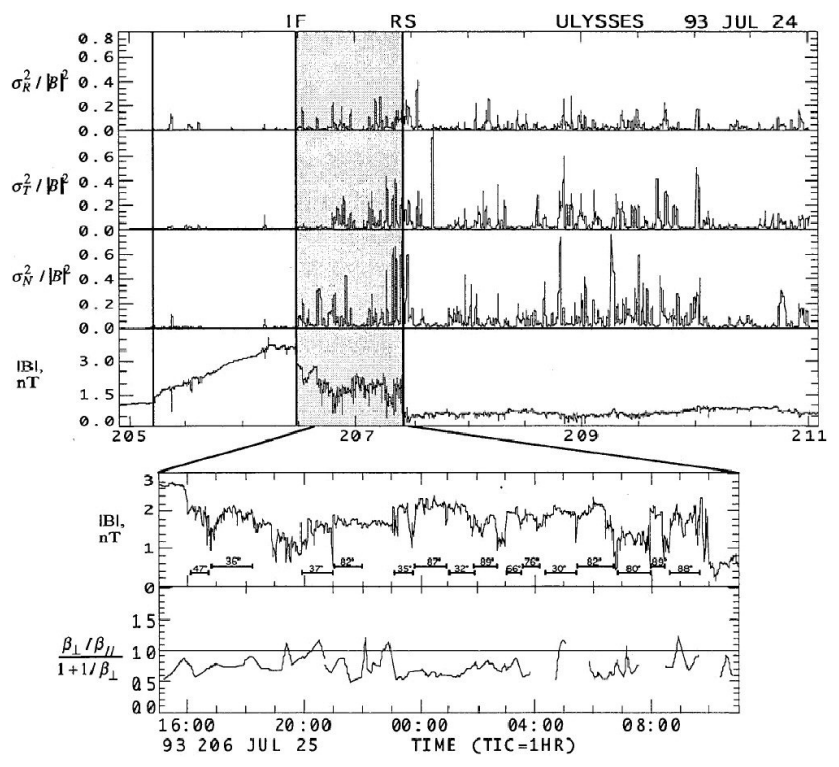

Fig. 14. Normalized one min variances for the trailing portion of a CIR. Intervals of Alfvén waves were studied using the minimum variance technique. The intervals are indicated by bars in the inset and the wave direction of propagation given.

shock compression. Wave-wave interactions may lead to the production of other wave modes as well.

Figure 14 is an example of a CIR and the field variances. The top panel shows the normalized (by $1 / B^{2}$ ) 1 min field variances for the magnetic field in a RTN coordinate system. It can be noted that the normalized variances in the latter part of the CIR (shaded region) are as large or larger than in the high speed stream proper. There are very low variances in the first part of the CIR and in the slow speed stream upstream of the forward shock (the latter is denoted by a vertical line at 06:00 UT day 205). The bottom panel of the top section of the figure gives the magnetic field magnitude. There are many MDs present in the latter part of the CIR. There are only a few MDs in the leading part of the CIR.

The lower insert gives an expanded view of the magnetic field for the trailing part of the CIR. The MHs/MDs are more readily apparent here. Small sections of this trailing part of the CIR were analyzed using the minimum variance technique. The intervals analyzed exclude the MHs/MDs proper. The purpose of the effort was to determine the polarization and angle of propagation of the Alfvén waves present. The intervals of analyses and the resultant $\theta_{k B}$ values are given in the figure. It was found that the waves are elliptically polarized and were propagating at large angles to the ambient field direction.

It should be noted that if one does a cross-correlation analyses between the magnetic field components and the velocity components, the region of lowest correlation coefficient will be the latter half of the CIR (see Tsurutani et al., 1995b). The reason for this is the presence of the many MHs/MDs. The low correlation coefficient however cannot simply be interpreted as a lack of Alfvén waves, as was shown by the

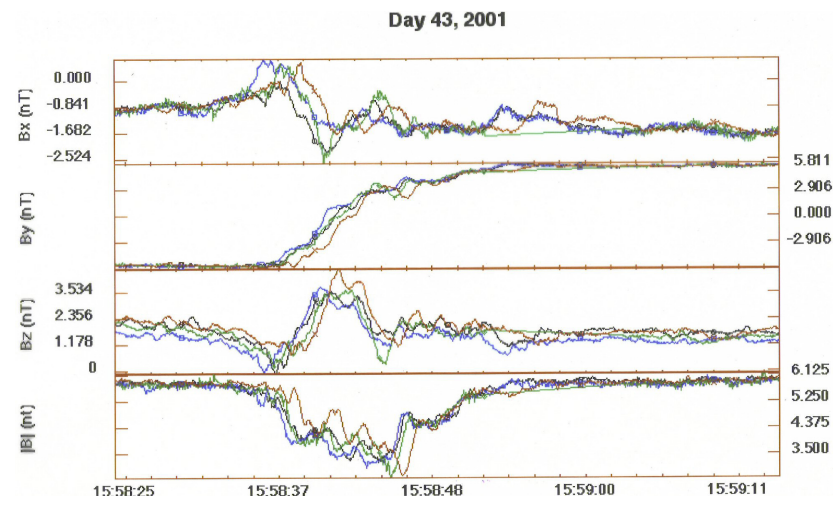

Fig. 15. A MD detected by all four CLUSTER spacecraft. The MD is at the edge of a phase-steepened Alfvén wave.

minimum variance analyses. If the analyses are performed over the intervals excluding the MHs/MDs, the coefficients will be high.

\subsection{Rate of Alfvén wave phase-steepening}

The rate of Alfvén wave phase-steepening can be determined by making two-point measurements separated by space and time. For this purpose we use the CLUSTER and ACE spacecraft magnetometer data. The four CLUSTER spacecraft are in orbit around the Earth. The ACE spacecraft is in orbit about the $L_{1}$ libration point, $0.01 \mathrm{AU}$ upstream of the Earth. Thus the solar wind first passes ACE and then sometime later ( $\sim 30 \mathrm{~min}$ to $1 \mathrm{~h}$, depending on the solar wind speed), passes the CLUSTER spacecraft. In the solar wind, the Alfvén wave phase speed is typically $1 / 5$ to $1 / 10$ the solar wind speed, so the waves are primarily convected past the spacecraft.

Figure 15 gives CLUSTER magnetic field observations of a MD in RTN coordinates. This interplanetary discontinuity/MD was identified by Knetter et al. (2004). Care was taken to avoid contamination by foreshock waves. The MD is present at $\sim 15: 58: 40 \mathrm{UT}$ for all four satellites. It is located at the edge of a phase-steepened Alfvén wave (the Alfvén wave is not shown). A large discontinuity is noted in the $B_{T}$ component, from $\sim 15: 58: 37$ UT to $\sim 15: 58: 52$ UT. The discontinuity spans the same temporal interval as the field decrease of the MD.

The same discontinuity has been identified at ACE, shown in Fig. 16. The solar wind speed at CLUSTER and the distance from CLUSTER to ACE was used to calculate when the discontinuity should have occurred at ACE. The detection of the ACE MD was within $10 \mathrm{~s}$ of the calculated time. Here the MD spans the interval between 15:01:49 and 15:02:20 UT. The thickness is $31 \mathrm{~s}$, while at CLUSTER the MD is $\sim 15 \mathrm{~s}$. The MD is thinner at CLUSTER.

Table 1 gives the 1/e MD dimensions of seven MDs at CLUSTER and the corresponding values at ACE during 2001. For one CLUSTER event, we were unable to identify a corresponding ACE event (the event on day 33-34). For all 6 remaining cases where the MD was identified at ACE, it was 
Table 1. The magnitude of the MD magnetic field magnitude decreases and the MD durations. The table shows the seven MDs in 2001 measured both at CLUSTER and ACE.

\begin{tabular}{ccccc}
\hline Event & \multicolumn{2}{c}{$\begin{array}{c}\text { Magnetic field } \\
\text { decrease }(\mathrm{nT})\end{array}$} & \multicolumn{2}{c}{ Decrease duration (s) } \\
& CLUSTER & ACE & CLUSTER & ACE \\
\hline $34(33)$ & Not clear & N/A & Not clear & N/A \\
43 & $\sim 2.77$ & $\sim 2.78$ & $\sim 8$ & $\sim 35$ \\
50 & $\sim 1.5$ & $\sim 0.57$ & $\sim 6$ & $\sim 38$ \\
51 (a) & $\sim 1.68$ & $\sim 1.46$ & $\sim 2.6$ & $\sim 13$ \\
51 (b) & $\sim 2.58$ & $\sim 1.97$ & $\sim 3.3$ & $\sim 48$ \\
$77(76)$ & $\sim 4.58$ & $\sim 0.91$ & $\sim 0.75$ & $\sim 16$ \\
77 & $\sim 1.20$ & $\sim 2.65$ & $\sim 8.3$ & $\sim 46$ \\
\hline
\end{tabular}

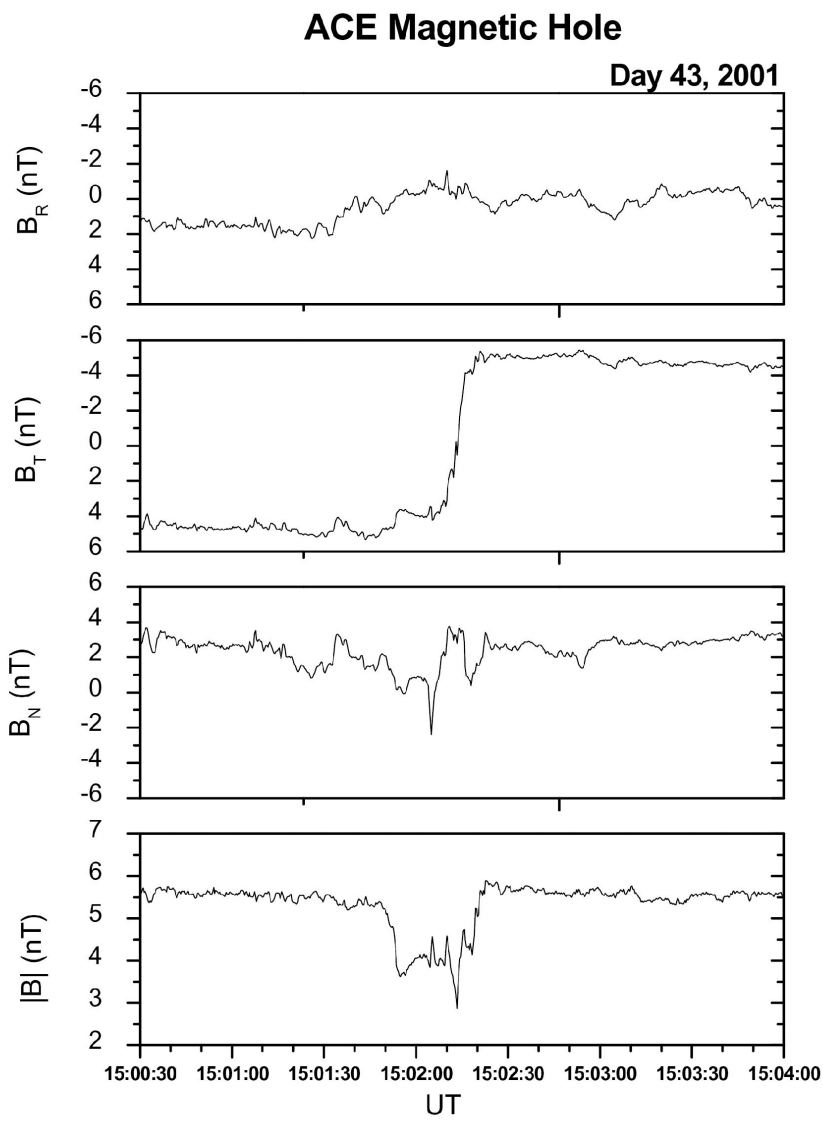

Fig. 16. The same MD in Fig. 15, but detected earlier by ACE, $\sim 0.01$ AU upstream of Cluster.

found that the MD was thinner at CLUSTER than at ACE. The ratio of $T_{\mathrm{ACE}} / T_{\mathrm{CLUSTER}}$ was a minimum of 4.4 on day 43 to a maximum of 21.3 on day 76-77. For further calculations we will use a conservative value of $T_{\mathrm{ACE}} / T_{\mathrm{CLUSTER}}=5$.

From the above, a steepening factor of $\sim 5$ will be used for calculations of steepening rates. As previously mentioned, the solar wind speed is typically a factor of 5 to 10 times (say an average of $~ 7$ ) the local Alfvén speed. Thus for an Alfvén wave with wavelength $\lambda_{r} \approx 0.01 \mathrm{AU}$, the wave has propagated

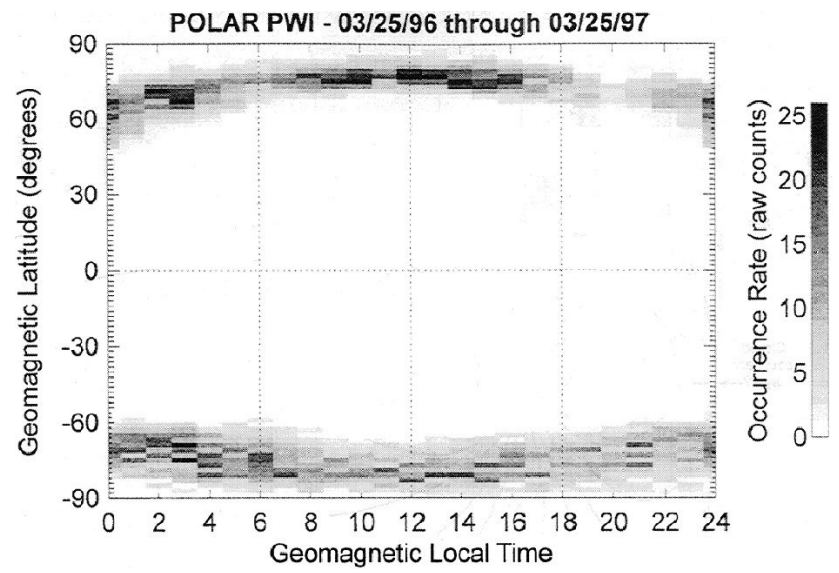

Fig. 17. The magnetic latitude-local time dependence of polar cap boundary layer waves. The wave latitude dependence is the same as the auroral oval. It is concluded that the boundary layer is on closed magnetic field lines that connect to the auroral oval. The figure is taken from Tsurutani et al. (2001).

only 1/7 $\lambda_{r}$ between ACE and CLUSTER. The wave has been primarily convected across the distance. Stated another way, the Alfvén waves have steepened a factor of $\sim 5$ times while propagating $1 / 7 \lambda_{r}$. Thus, the Alfvén waves are steepening a factor of $\sim 35$ while propagating one wavelength relative to the solar wind plasma.

\subsection{Alfvén waves in the magnetopause boundary layer}

At the very center edge of the Earth's magnetosphere, there is a plasma boundary layer. It is present almost all ( $95 \%)$ of the time. The POLAR ELF/VLF plasma wave instrument was used to find the latitude - local time dependence of the emissions. One year of data was analyzed for this purpose. The results of the survey is shown in Fig. 17. Both northern and southern hemispheric data are shown.

The ELF/VLF plasma wave location map out a continuous oval. The waves are detected at $\sim 75^{\circ}$ magnetic latitude at local noon and $\sim 65^{\circ}$ magnetic latitude at local midnight. This is the same distribution as the auroral oval (Feldstein and Starkov, 1970). Thus it is assumed that the Polar Cap Boundary Layer (PCBL) waves are detected on auroral zone magnetic field lines.

Alfvén waves have been detected at local noon near this boundary region near the polar cusp. Two examples of these waves are shown in Fig. 18 in minimum variance coordinates. Note the presence of MDs within the magnetosphere. The waves are found to be elliptically polarized, $\lambda_{1} / \lambda_{2}=4.5$ and 4.3 , and propagating obliquely to the ambient magnetic field, $\theta_{k B}=77^{\circ}$ and $69^{\circ}$, respectively.

Extremely large amplitude electromagnetic proton cyclotron waves were detected during the first Alfvén wave/MD event. This is shown in Fig. 19. The three orthogonal components of the wave electric and wave magnetic field are given in field-aligned (and orthogonal) coordinates. The magnetic oscillations are noted in not only the 


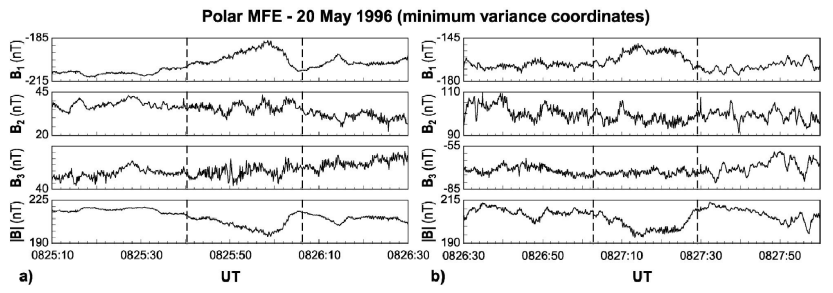

Fig. 18. Alfvén waves in the PC boundary layer. The waves are propagating obliquely to the ambient magnetic field. There are large MDs associated with the waves.

two orthogonal magnetic components, but also in the parallel component, implying wave propagation across the ambient magnetic field. Minimum variance analyses indicate that these waves were propagating at $70^{\circ}$ relative to the ambient magnetic field. These waves have a peak-to-peak amplitude of $\sim 14 \mathrm{nT}$ and are perhaps the largest amplitude proton cyclotron waves ever detected in the Earth's magnetosphere.

Another type of wave mode identified during these Alfvén wave intervals are electric bipolar pulses, also called electron holes. Figure 20 shows the onset of an electron hole wave train out of thermal background. Another such event was noted and reported from the second Alfvén wave interval.

A source of instability for the waves was sought. The electrons were found to be isotropic in distribution (Tsurutani et al., 2003). There was no evidence of bi-streaming electrons, the typical method of generating electron holes in computer simulations (Omura, 1996; Goldman et al., 1999; Singh, 2003). Another possibility has been suggested, that of generating short duration counter-streaming electrons by the electric fields associated with either the obliquely propagating electromagnetic cyclotron waves or the Alfvén waves. A schematic is given in Fig. 21. Lakhina et al. (2003; 2004, 2005) have proposed that nonlinear low-frequency waves, consisting of Alfvén and/or proton cyclotron waves, can drive high-frequency electrostatic modes. The electrostatic modes saturate by trapping electrons, thereby leading to the generation of electron holes. The free energy sources for these electrostatic instabilities are the strong transient parallel currents, which are produced due to relative motion of electrons and ions in the presence of large amplitude lowfrequency waves. The calculated growth rates and wave saturation time scales are found to be consistent with the observations.

\section{Summary and comments}

Nonlinear Alfvén waves are found to phase-steepen in interplanetary space. The waves are therefore dispersive, forming a high-frequency component at their leading edges. Some property of the leading edges of the waves cause perpendicular ion and electron heating. Thus the Alfvén waves are dissipative as well. These two properties, dispersion and dissipation, are common to all shocks. A steady state is reached when the dispersive and dissipative effects are bal-

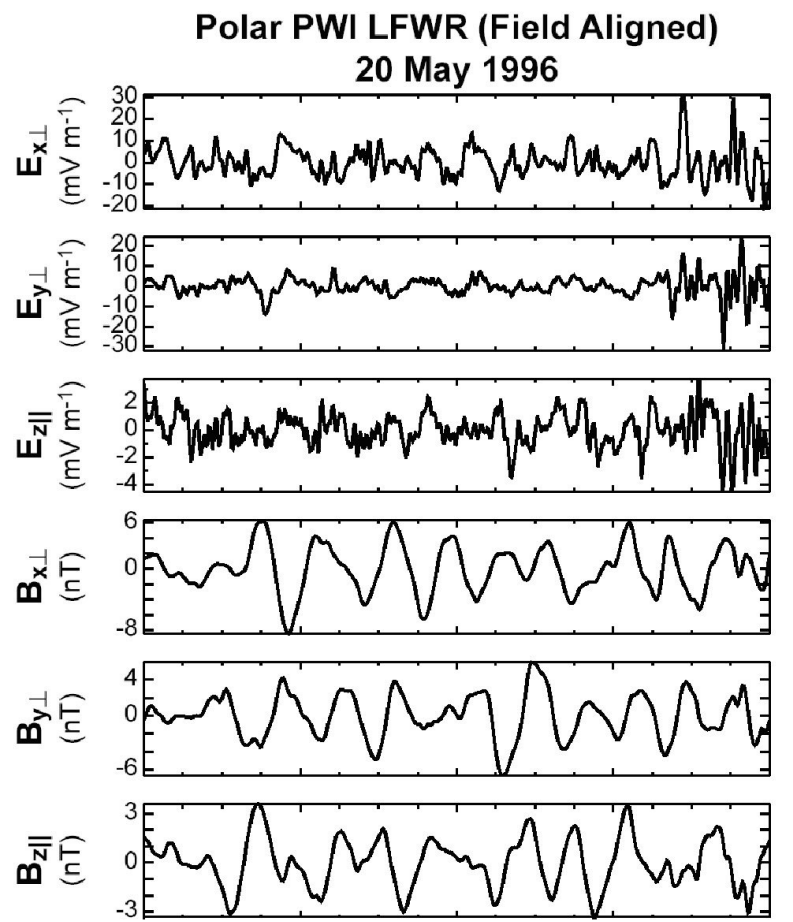

08:25:57.853 08:25:59.013 08:26:00.173 $08: 26: 01.333 \quad 08: 26: 02.493$

SCET (UT) University of lowa

\begin{tabular}{|cc|}
\hline$R_{E}=6.05$ & \\
$L=27.32$ & $M L T=11.62$ \\
$\lambda_{m}=61.96$ & $F_{c p}=3.05 \mathrm{~Hz}$ \\
\hline
\end{tabular}

Fig. 19. Large amplitude proton cyclotron waves associated with the Alfvén waves in Fig. 18. These electromagnetic waves are propagating across the ambient magnetic field.

anced. However, for the limited events that were examined using the CLUSTER-ACE data sets, phase-steepening was still occurring at $1 \mathrm{AU}$. Whether this was a chance occurrence or not is unknown at this time. In any case, the study of nonlinear Alfvén waves may be an excellent chance to identify the plasma properties of intermediate shocks.

The wave phase-steepening process and dissipative effects lead to an evolving "turbulence" that is difficult to describe and perhaps even more difficult to analyze using standard techniques. For interplanetary space, the Alfvén waves develop high frequency electromagnetic components through nonlinear steepening. The dissipation of these steepened edges into proton perpendicular kinetic energy leads to nonpropagating (convective) magnetic decreases and magnetic holes. Instabilities associated with proton acceleration leads to the growth of propagating proton cyclotron waves and nonpropagating mirror mode structures. In the Earth's magnetosphere, proton cyclotron waves are believed to become damped and through this process create electron holes. There are also higher frequency waves generated by electron instabilities as well. Thus it is believed that the Alfvén waves dissipate into propagating waves at both high and low 


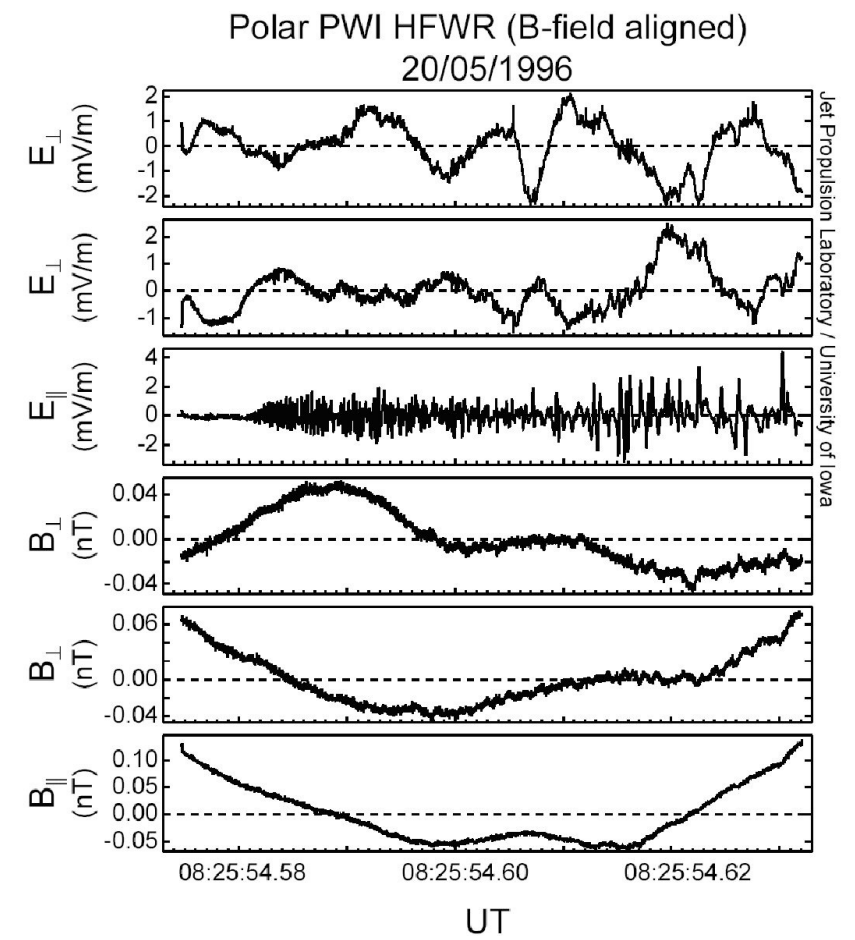

Fig. 20. A bipolar pulse (electron hole) event onset. This event occurred during the large amplitude proton cyclotron waves of Fig. 19. Taken from Tsurutani et al. (2001).

frequencies and also nonpropagating structures. The nonpropagating structures vary from small scale $\sim 2-3 r_{p}$ (MHs), to medium scale (mirror mode $\sim 20-40 r_{p}$ ) to large scales (MDs). Further complications are that this "turbulence" will most likely not be stationary in time. We refer the reader to Chang et al. (2004) for a discussion of magnetospheric "intermittent turbulence" and Lakhina et al. (2000) for a detailed review of wave modes present in the PCBL.

\subsection{Compressive structures}

The MDs in interplanetary space and the magnetosphere are currently thought to be static, nonpropagating structures, to first order. If they are found to be bounded by slow shocks, this picture will have to be revised. Slow shocks are propagating structures and will have different dissipation mechanisms as well.

Care should be taken when interpreting power spectra of interplanetary magnetic fields. Much of the "power" both in the field components and field magnitude may be related to the MDs/MHs. Additionally, it was indicated earlier in this paper that there were broad regions of small field enhancements in between the MHs/MDs. If indeed the MDs/MHs are created by the diamagnetic effect (displaced magnetic fields) of the proton perpendicular kinetic energy, the positive field enhancements may be those displaced fields. These field enhancements would therefore also not be propagating structures.

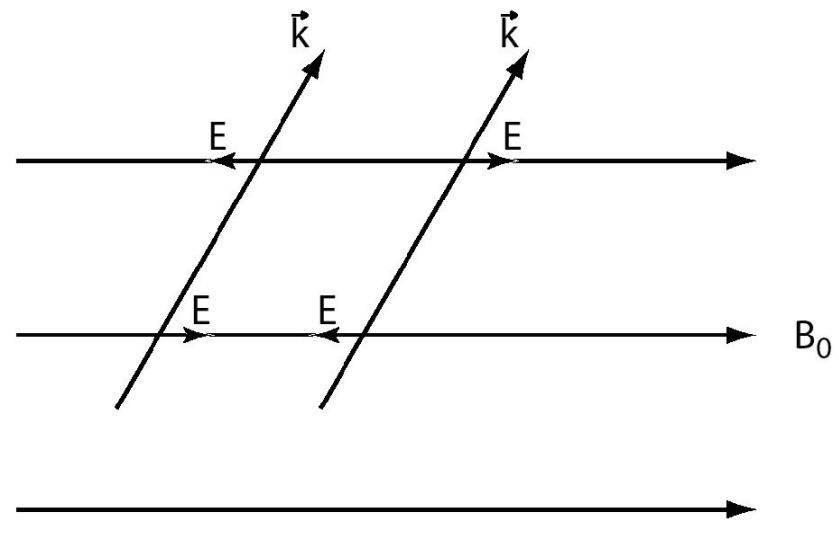

Fig. 21. A schematic of cross-field propagation of proton cyclotron waves. The electric fields from the waves can accelerate electrons so they form unstable bi-directional distributions (Lakhina et al., 2003).

\subsection{Particle scattering}

Charged particle scattering by cyclotron and Landau resonant interactions with propagating electromagnetic and electrostatic waves is a well developed field (Kennel and Petschek, 1966; Tsurutani and Lakhina, 1997). All of the theories developed for these interactions assume constant ambient magnetic field magnitudes. However, nonresonant interactions with structures that have either magnetic field magnitude decreases or increases can also scatter charged particles. This topic is far less developed and would be an interesting area for young enterprising scientists. One facet of these interactions has been briefly looked at, cross-field diffusion of energetic 1 to $10 \mathrm{MeV}$ protons (Tsurutani et al., 1999; Tsurutani and Lakhina, 2000). The basic interaction is akin to a gradient drift mechanism, but with the breaking of the first adiabatic invariant. Using distributions of MD properties (MD thicknesses, etc.), a rough estimate of a cross-field diffusion rate of $\sim 0.1 D_{\text {Bohm }}$ was estimated. For more precise values, Monte Carlo analyses would be appropriate.

A mechanism has been sought for the energization of Jovian magnetospheric energetic particles. Nishida (1976) has suggested a particle "circulation" model when the particles radially diffuse from the outer parts of the magnetosphere to the inner parts, gaining energy in the process. In this scenario, the particles then scatter across the magnetic field near their low-altitude mirror points to larger L-shell magnetic field lines and then inwardly diffuse once more. Fujimoto and Nishida (1990) have examined this process for recirculating $\mathrm{MeV}$ electrons in the Earth's magnetosphere. They find that if several tens of $\mathrm{mV} / \mathrm{m}$ electric fields at $\sim 1 \mathrm{~s}$ are present at low altitudes, particle recirculation can occur and some of the measured particle features can be duplicated. Another possibility that has not been examined are nonresonant interactions with magnetic decrease structures (or plasma cavities associated with double layers) at middle or low altitudes. 
Although no serious thought has been applied to the idea of nonresonant interactions for the acceleration of relativisitic ("killer") electrons (Paulikas and Blake, 1979; Baker et al., 1994; Horne et al., 2003), this mechanism may prove to be important for this physical process as well.

\subsection{Alfvén wave-MD interactions}

In the scenario proposed above, some mechanism (the Ponderomotive Force?) leads to the perpendicular acceleration of particles, which in turn causes the formation of MDs by a diamagnetic effect. Within these high beta MDs, the phase velocity of Alfvén waves are lowered. Thus Alfvén waves propagating through MDs will proceed considerably more slowly. How will this affect the dissipation rate of the Alfvén waves? If more Alfvén waves "collide" with the MDs, will this enlarge the MDs further? Computer simulation studies of Alfvén wave-MD interactions will help provide some of the answers.

\subsection{The ponderomotive force}

The proposed mechanism for the particle perpendicular acceleration is the Ponderomotive Force associated with the wave field gradient. Detailed discussions can be found in Tsurutani et al. (2002b) and Dasgupta et al. (2003). This is a nonresonant interaction that occurs at frequencies well away from cyclotron resonance. There are other possible mechanisms as well and we are currently investigating their particle acceleration efficiencies. One such possibility is the damping of proton cyclotron waves. The phase-steepened edges of Alfvén waves contains a lot of high frequency wave power, and some of it will be at the proton cyclotron frequency. Whether this power is sufficient or not to give the necessary acceleration to protons is not known at this time. This scenario will also require a different mechanism to accelerate electrons. Another possibility is lower hybrid wave heating which could accelerate and heat ions in the perpendicular direction and the electrons in the parallel direction.

\section{Final comments}

This paper attempts to review Alfvénic coherent structures in space plasmas. We have approached the topic from the point of view of observations and their implications. We hope that they will provide an interesting and reliable source of information for comparison to forthcoming theories and numerical simulations. The need for better theoretical understanding is hopefully obvious. One example is the wave phase-steepening problem. One of the original works on this topic was by Cohen and Kulsrud (1974) using an MHD analysis. When it was discovered that cometary magnetosonic (fast mode) waves were noted to be phase-steepened, Tsurutani et al. (1987) showed that MHD theory gave qualitative (but not quantitative) agreement with the observations. The lack of taking finite Larmor radius effects into account is an obvious omission. Matsumoto and Nagai (1981) performed a one-and-two half dimensional magnetostatic particle simulation assuming perpendicularly propagating magnetosonic waves. Many of the features of the waves were noted in the results. More recent theoretical work on the evolution of nonlinear Alfvén waves have been performed by Medvedev and Diamond (1996), Medvedev et al. (1997), Goldstein et al. (1999), Vasquez and Hollweg (1999, 2001), and Buti et al. $(1999,2001)$. These works were again able to show many of the observed features of the nonlinear waves, but also predicted many effects not found in the solar wind data. More recently, magnetic holes have been simulated by an interaction with Alfvén waves with the Earth's bow shock, but for only a specific magnetic field geometry (Tsubouchi and Matsumoto, 2005). From our observations, we also note that kinetic ions (and electrons?) are playing an important role. It is possible that it will be necessary to use a 3D particle code modeling waves with field gradients, to fully simulate the natural case. Some of the important questions are: what are the various processes that lead to Alfvén wave steepening? How fast does steepening occur as a function of plasma $\beta$ ? How is the steepening arrested, if it is? What dispersive and/or dissipation processes are at work? Although MDs/MHs can be created by the diamagnetic effect of perpendicularly heated plasma, what further evolution takes place such that the plasma density becomes greatly enhanced within these regions, as Winterhalter et al. (1994) has shown? These are a few of the interesting problems that exists for nonlinear Alfvén waves.

The overall picture of phase-steepened Alfvén waves is a complex one, particularly from the point of view of trying to understand their properties using standard data analysis techniques. Since the phase steepened edges (rotational in nature) will also have dissipation effects at the same location (compressional effects), analysis of these "discontinuities" will indicate mixed properties, perhaps leading to the confusion of their "types". This complex scenario also may explain why very few ideal rotational discontinuities $\left(B_{n} / B_{L}=1.0\right.$ and $\left.\Delta|B| / B=0\right)$ or ideal tangential discontinuities $\left(B_{n} / B_{L}=0\right)$ have been found.

The rapid evolution of the phase-steepened edges of Alfvén waves found in the ACE-CLUSTER data analyses indicates that wave dissipation is fairly rapid. However at this time, the amount of energy transfer from the waves to the solar wind plasma has not been calculated. This is a task that needs to be done. This rapid evolution of the waves explains why attempts at identifying the same discontinuity at two radially aligned spacecraft with longer spatial separations than 0.01 AU (Pioneer 10 and 11, Voyagers 1 and 2) have failed.

Acknowledgements. Portions of this work were done at the Jet Propulsion Laboratory, California Institute of Technology under contract with NASA. The Cluster portion of the work done at JPL was performed under a NASA Goddard Space Flight Center grant. Work at the University of Iowa was carried out under NASA Goddard Space Flight Center grants under the Polar and Cluster projects. 
Edited by: P.-L. Sulem

Reviewed by: one referee

\section{References}

Baker, D. N., Blake, J. B., Callis, L. B., Cummings, J. R., Hovestadt, D., Kanekal, S., Blecker, B., Mewaldt, R. A., and Zwickl, R. D.: Relativistic electron acceleration and decay timescales in the inner and outer radiation belts: SAMPEX, Geophys. Res. Lett., 21, 409-412, 1994.

Balogh, A., Gosling, J. T., Jokipii, J. R., Kallenbach, R., and Kunow, H. (Eds): Corotating Interaction Regions, Space Sci. Rev. 89, 2002.

Bame, S. J., Asbridge, J. R., Feldman, W. C., Montgomery, M. D., and Gary, S. P.: Evidence for local ion heating in the solar wind high speed stream, Geophys. Res. Lett., 2, 373-375, 1975.

Baumgärtel, K.: Soliton approach to magnetic holes, J. Geophys. Res., 104, 28 295-28 308, 1999.

Belcher, J. W. and Davis Jr., L.: Large-amplitude Alfvén waves in the interplanetary medium, 2, J. Geophys. Res., 76, 3534-3563, 1971.

Belcher, J. W. and Solodyna, C. V.: Alfvén waves and directional discontinuities in the interplanetary medium, J. Geophys. Res., 80, 181-186, 1975.

Bhattacharjee, A., Ng, C. S, and Spangler, S. R.: Weakly compressible magnetohydrodynamic turbulence in the solar wind and the interstellar medium, Astrophys. J., 494, 409-418, 1998.

Bhattacharjee, A.,. Ng, C. S., Ghosh, S., and Goldstein, M. L.: A comparative study of four-field and fully compressible magnetohydrodynamic turbulence in the solar wind, J. Geophys. Res., 104, 24 835-24 844, 1999.

Burlaga, L. F., Lemaire, J. F., and Turner, J. M.: Interplanetary current sheets at 1 AU, J. Geophys. Res., 82, 3191-3200, 1977.

Burlaga, L. F.: Directional discontinuities in the interplanetary magnetic field, Sol. Phys., 7, 54-71, 1969.

Buti, B., Galinski, V. L., Shapiro, V. I., Lakhina, G. S., Tsurutani, B. T., Diamond, P., and Medvedev, M. V.: Evolution of nonlinear Alfvén waves in streaming inhomogeneous plasmas, Astrophys. J., 523, 849-854, 1999.

Buti, B., Tsurutani, B. T., Neugebauer, M., and Goldstein, B. E.: Generation mechanism for magnetic holes in the solar wind, Geophys. Res. Lett., 28, 1355-1358, 2001.

Chang, T., Tam, S. W. Y., and Wu, C.-C.: Complexity induced anisotropic bimodal intermittent turbulence in space plasmas, Phys. of Plasmas, 11, 1287-1299, 2004.

Cohen, R. H. and Kulsrud, R. M.: Nonlinear evolution of parallel propagating hydromagnetic waves, Phys. Fluids, 17, 2215-2225, 1974.

Colburn, D. S. and Sonett, C. P.: Discontinuities in the solar wind, Space Sci. Rev., 5, 439-506, 1966.

Coleman, P. J.: Turbulence, viscosity and dissipation in the solar wind plasma, Astrophys. J., 153, 371-388, 1968.

Dasgupta, B., Tsurutani, B. T., and Janaki, M. S.: A kinetic approach to the Ponderomotive Force, Geophys. Res. Lett., doi:10.1029/2003GL017385, 2003.

Denskat, K. U., Neubauer, F. M., and Schwenn, R.: Properties of "Alfvénic" fluctuations near the sun: Helios-1 and Helios-2, in Solar Wind Four, edited by: Rosenbauer, H., 392, 1981.

Fairfield, D. H.: Bow shock associated waves observed in the far upstream interplanetary medium, J. Geophys. Res., 74, 35413553, 1969.
Farrugia, C. J., Vasquez, B., Richardson, I. G., et al.: A reconnection layer associated with a magnetic cloud, Adv. Space Res., 28, 759-764, 2001.

Feldstein, Y. I. and G. V. Starkov: The auroral oval and the boundary of closed field lines of geomagnetic field, Planet. Space. Sci., 18, 501-508, 1970.

Fränz, M., Burgess, D., and Horbury, T. S.: Magnetic depressions in the solar wind, J. Geophys. Res., 105, 12725-12732, 2000.

Fujimoto, M. and Nishida, A.: Energization and anisotropization of energetic electrons in the Earth's radiation belt by the recirculation process, J. Geophys. Res., 95, 4265-4270, 1990.

Glassmeier, K.-H., and Neubauer, F. M.: Low-frequency electromagnetic plasma waves at comet P/Grigg-Skjellerup: Overview and spectral characteristics, J. Geophys. Res., 98, 20 921-20 935, 1993.

Goldman, M. V., Openheim, M. M., and Newman, D. L.: Nonlinear two-stream instabilities as an explanation for auroral bipolar wave structures, Geophys. Res. Lett., 26, 1821-1824, 1999.

Goldstein, M. L., Robers, D. A., and Matthaeus, W. H.: Magnetohydrodynamic turbulence in the solar wind, Ann. Rev. Astron. Astrophys., 33, 283-326, 1995.

Goldstein, M. L., Roberts, D. A., Deane, A. E., Ghosh, S., and Wong, H. K.: Numerical simulations of Alfvénic turbulence in the solar wind, J. Geophys. Res., 104, 14 437-14 452, 1999.

Ho, C. M., Tsurutani, B. T., Lin, N., Lanzerotti, L. J., Smith, E. J., Goldstein, B. E., Buti, B., Lakhina, G. S., and Zhou, X.-Y.: A pair of forward and reverse slow-mode shocks detected by Ulysses at 5 AU, Geophys. Res. Lett., 25, 2613-2616, 1998.

Hollweg, J. V.: Cyclotron resonance in corona holes: Heating and acceleration of protons, $\mathrm{O}^{5+}, \mathrm{Mg}^{9+}$, J. Geophys. Res., 104, 24 781-24 792, 1999.

Hoppe, M. M., Russell, C. T., Frank, L. A., Eastman, T. E., and Greenstadt, E. W.: Upstream hydromagnetic waves and their association with backstreaming ion populations: ISEE 1 and 2 observations, J. Geophys. Res., 86, 4471-4492, 1981.

Horbury, T. S., Burgess, D., and Franz, M.: Three spacecraft observations of solar wind discontinuities, Geophys. Res. Lett., 28, 677, 2001.

Horbury, T. S. and Balogh, A.: Evolution of magnetic field fluctuations in high speed solar wind streams: Ulysses and Helios observations, J. Geophys. Res., 106, 15 929-15 940, 2001.

Horne, R. B., Meredith, N. P., Thorne, R. M., Heynderickx, D., Iles, R. H. A., and Anderson, R. R.: Evolution of energetic electron pitch angle distributions during storm time electron acceleration to $\mathrm{MeV}$ energies, J. Geophys. Res., 108, doi:10.1029/2002JA009468, 2003.

Kennel, C. F. and Petschek, H. F.: Limit on stably trapped particle fluxes, J. Geophys. Res., 71, 1-28, 1966.

Knetter, T., Neubauer, F. M., Horbury, T., and Balogh, A.: Four-point discontinuity observations using Cluster magnetic field data: A statistical survey, J. Geophys. Res., 109, doi:10.1029/2003JA010099, 2004.

Lakhina, G. S., Tsurutani, B. T., Kojima, H., and Matsumoto, H.: "Broadband" plasma waves in the boundary layers, J. Geophys. Res., 105, 27 791-27 832, 2000.

Lakhina, G. S., Tsurutani, B. T., Singh, S. V., and Reddy, R. V.: Some theoretical models for solitary structures of boundary layer waves, Nonlin. Proc. Geophys., 10, 65-73, 2003,

SRef-ID: 1607-7946/npg/2003-10-65.

Lakhina, G. S., Tsurutani, B. T., and Pickett, J. S.: Association of Alfvén waves and proton cyclotron waves with electrostatic bipolar pulses: Magnetic hole events observed by Polar, Nonlin. 
Proc. Geophys., 11, 205-213, 2004,

SRef-ID: 1607-7946/npg/2004-11-205.

Lakhina, G. S., Tsurutani, B. T., and Pickett, J. S.: Generation of electric solitary structures (electron holes) by nonlinear lowfrequency waves, Physica Scripta, in press, 2005.

Landau, L. D. and Lifshitz, E. M.: Electrodynamics of Continuous Media, Pergamon, New York, 255, 1960.

Lee, N. C. and Parks, G. K.: Ponderomotive force in a warm twofluid plasma, Phys. Fluids, 26, 724-729, 1983.

Lepping, R. P. and Behannon, K. W.: Magnetic field directional discontinuities: Characteristics between 0.46 and 1.0 AU, J. Geophys. Res., 91, 8725-8741, 1986.

Li, X. L. and Temerin, M.: Ponderomotive effects on ion acceleration in the auroral zone, Geophys. Res. Lett., 20, 13-16, 1993.

Lin, N., Kellogg, P. J., MacDowall, R. J., Balogh, A., Forsyth, R. J., Phillips, J. L., Buttighoffer, A., and Pick, M.: Observations of plasma waves in magnetic holes, Geophys. Res. Lett., 22, $3417-$ 3420, 1995.

Lin, N., Kellogg, P. J., MacDowall, R. J., Tsurutani, B. T., and. Ho, C. M.: Langmuir waves associated with discontinuities in the solar wind: A statistical study, Astron. Astrophys., 316, 425429, 1996

Luhr, H. and Klocker, N.: AMPTE-IRM observations of magnetic cavities near the magnetopause, Geophys. Res. Lett., 14, 186189, 1987.

MacDowall, R. J., Lin, N., Kellogg, P. J., Balogh, A., Forsyth, R. J., and Neugebauer, M.: Langmuir waves in magnetic holes: Source mechanism and consequences, Proc. Eighth Intl. Sol. Wind Conf., edited by: Winterhalter D. et al., Am. Inst. Phys., 301, 1996.

Marsch, E, Schwenn, R., Rosenbauer, H., Muehlhaeuser, K.-H., Pilipp, W., and Neubauer, F. M..: Solar wind protons: Threedimensional velocity distributions and derived plasma parameters measured between 0.3 and 1 AU, J. Geophys. Res., 87, 5272, 1982.

Matsumoto, H., and Nagai, JK.: Steepening, soliton and Landau damping of large-amplitude magnetosonic waves: Particle code computer simulation, J. Geophys. Res., 86, $10068-10$ 072, 1981.

Matthaeus, W. H., Bieber, J., and Zank, G. P.: Unquiet on any frontanisotropic turbulence in the solar wind, Rev. Geophys., 33, 609, 1995.

Mazelle, C., Reme, H., Neubauer, F.M., and Glassmeier, K.-H.: Comparison of the main magnetic field and plasma features in the environment of comets Grigg-Skjellerup and Halley, Adv. Space Res., 16, 41-45, 1995.

McComas, D. J., Elliott, H. A., Gosling, J. T., Reisenfeld, D. B., Skoug, R. M., Goldstein, B. E., Neugebauer, M., and Balogh, A.: Ulysse's second fast-latitude scan: Complexity near solar maximum and the reformation of polar coronal holes, Geophys. Res. Lett., 29, doi:10.1029/2001GL014164, 2002.

Medvedev, M. V. and Diamond, P. H.: Fluid models for kinetic effects on coherent nonlinear Alfvén waves, I. Fundamental theory, Phys. Plasmas, 3, 863-873, 1996.

Medvedev, M. V., Shevchenko, V. I., Diamond, P. H., and Galinsky, V. L.: Fluid models for kinetic effects on coherent nonlinear Alfvén waves, II. Numerical solutions, Phys. Plasmas, 4, $1257-$ $1285,1997$.

Ness, N. F., Scearce, C. S., and Canarano, S.: Preliminary results from the Pioneer 6 magnetic field experiment, J. Geophys. Res., 71, 3305, 1966.

Neubauer, F. M. and Barnstorf, H.: Recent observational and theoretical results on discontinuities in the solar wind, Solar Wind
Four, Rep. MPAE-W-100-81-31, edited by: Rosenbauer, H. R., Max-Planck-Inst., Lindau, Germany, 1981.

Neubauer, F. M., Glassmeier, K.-H., Coates, A. J., and Johnstone, A. D.: Low-frequency electromagnetic plasma wave fields at comet P/Grigg-Skjellerup: Analysis and interpretation, J. Geophys. Res., 98, 20 937-20 953, 1993.

Neugebauer, M., Clay, D. R., Goldstein, B. E., Tsurutani, B. T., and Zwickl, R. D.: A reexamination of rotational and tangential discontinuities in the solar wind, J. Geophys. Res., 89, 53955408, 1984.

Neugebauer, M., Goldstein, B. E., Winterhalter, D., Smith, E. J., McDowell, R. J., and Gary, S. P.: Ion distribution in large magnetic holes in the fast solar wind, J. Geophys. Res., 106, 56355648, 2001.

Nishida, A.: Outward diffusion of energetic particles from the Jovian radiation belt, J. Geophys. Res., 81, 1771-1773, 1976.

Omura, Y., Matsumoto, H., Miyake, T., and Kojima, H.: Electron beam instabilities as generation mechanisms of electrostatic solitary waves in the magnetotail, J. Geophys. Res., 101, 2685-2698, 1996.

Paulikas, G. A. and Blake, J. B.: Effects of the solar wind on magnetospheric dynamics: Energetic electrons at the synchronous orbit, in Quantitative Modeling of Magnetospheric Processes, edited by Olsen, W. P., AGU Press, Wash. D.C., 21, 280, 1979.

Phillips, J. L., Bame, S. J., Feldman, W. C., Gosling, J. T., McComas, D. J., Goldstein, B. E., Neugebauer, M., and Hammond, C. M.: Ulysses solar wind plasma observations from peak southerly latitude through perihelion and beyond, in: Solar Wind Eight, edited by: Winterhalter, D., Gosling, J. T., Habbal, S. R., Kurth, W. S., and Neugebauer, M., AIP Conference Proc. 382, New York, 416-419, 1996.

Pizzo, V. J.: Interplanetary shocks on the large scale: A retrospective on the last decade's theoretical efforts, in Collisionless Shocks in the Heliosphere: Reviews of Current Research, edited by Stone, R. G. and Tsurutani, B. T., AGU Press, Washington D.C., 35, 51-68, 1985.

Russell, C. T., Childers, D. D., and Coleman Jr., P. J.: Ogo 5 observations of upstream waves in the interplanetary medium: Discrete wave packets, J. Geophys. Res., 76, 845-861, 1971.

Russell, C. T. and Elphic, R. C.: Initial ISEE magnetometer results: Magnetic observations, Space Sci. Rev., 22, 681-715, 1978.

Singh, N.: Space-time evolution of electron-beam driven electron holes and their effects on the plasma, Nonl. Proc. Geophys., 10, 53-63, 2003,

\section{SRef-ID: 1607-7946/npg/2003-10-53}

Siscoe, G. L., Davis Jr., L., Coleman Jr., P. J., Smith, E. J., and Jones, D. E.: Power spectra and discontinuities of the interplanetary magnetic field: Mariner 4, J. Geophys. Res., 73, 61-82, 1968.

Smith, E. J.: Identification of interplanetary tangential and rotational discontinuities, J. Geophys. Res., 78, 2054-2063, 1973a.

Smith, E. J.: Observed properties of interplanetary rotational discontinuities, J. Geophys. Res., 78, 2088-2093, 1973 b.

Smith, E. J. and Wolf, J. H.: Observations of interaction regions and corotating shocks between one and five AU: Pioneers 10 and 11, Geophys. Res. Lett., 3, 137-140, 1976.

Smith, E. J. and Tsurutani, B. T.: Magnetosheath lion roars, J. Geophys. Res., 81, 2261-2266, 1976.

Smith, E. J., Balogh, A., Neugebauer, M., and McComas, D.: Ulysses observations of Alfven waves in the southern and northern solar hemispheres, Geophys. Res. Lett., 22, 3381-3384, 1995. 
Sonnerup B. U. and Cahill Jr., L. J.: Magnetopause structure and attitude from Explorer 12 observations, J. Geophys. Res., 72, 171, 1967.

Stasiewicz, K., Seyler, C. E., Mozer, F. S., Gustafsson, G., Pickett, J. S., and Popielawska, B.: Magnetic bubbles and kinetic Alfvén waves in the high-latitude magnetopause boundary, J. Geophys. Res., 106, 29 503-29 514, 2001.

Sugiura, M., Skillman, T. L., Ledley, B., and Heppner, J. P.: "Holes" in the magnetic field near the magnetopause, Eos Trans. AGU, 50, 278, 1969.

Treumann, R. A., Bostrom, L., LaBelle, J., and Sckopke, N.: The plasma wave signature of a "Magnetic Hole" in the vicinity of the magnetopause, J. Geophys. Res., 95, 19 099-19 114, 1990.

Tsubouchi, K. and Matsumoto, H.: The effect of the upstream rotational field on the formation of the magnetic depression in the shock downstream, J. Geophys. Res., in press, 2005.

Tsurutani, B. T. and Smith, E. J.: Interplanetary discontinuities: Temporal variations and the radial gradients from 1 to $8.5 \mathrm{AU}$, J. Geophys. Res., 84, 2773-2787, 1979a.

Tsurutani, B. T., Thomas, B. T., Smith, E. J., and Wolfe, H.: Alfvén waves and interplanetary discontinuities at large heliospheric distances: Pioneer 10, 11, in Proceedings of the XVIIth IUGG General Assembly, edited by N. Fukushima, International Union of Geodesy and Geophysics, Paris, 1979b.

Tsurutani, B. T., Smith, E. J., Thorne, R. M., Gosling, J. T., and Matsumoto, H.: Steepened magnetosonic waves at comet Giacobini-Zinner, J. Geophys. Res., 92, 11 074-11 082, 1987.

Tsurutani, B. T., Smith, E. J., Buti, B., Matsumoto, H., and Brinca, A.: Discrete phase changes within nonlinear steepened magnetosonic waves: Comet Giacobini-Zinner, Geophys. Res. Lett., 17, 1817-1820, 1990.

Tsurutani, B. T., Ho, C. M., Smith, E. J., Neugebauer, M., Goldstein, B. E., Mok, J. S., Arballo, J. K., Balogh, A., Southwood, D. J., and Feldman, W. C.: The relationship between interplanetary discontinuities and Alfvén waves: Ulysses observations, Geophys. Res. Lett., 21, 2267-2270, 1994.

Tsurutani, B. T., Glassmeier, K.-H., and Neubauer, F. M.: An intercomparison of plasma turbulence at three comets: GriggSkjellerup, Giacobini-Zinner, and Halley, Geophys. Res. Lett., 22, 1149-1152, 1995a.

Tsurutani, B. T., Ho, C. M., Arballo, J. K., and Goldstein, B. E.: Large amplitude IMF fluctuations in corotating interaction regions: Ulysses at midlatitudes, Geophys. Res. Lett., 22, 33973400, 1995 b.

Tsurutani, B. T., Ho, C. M., Arballo, J. K., Smith, E. J., Goldstein, B. E., Neugebauer, M., Balogh, A., and Feldman, W. C.: Interplanetary discontinuities and Alfvén waves at high heliographic latitudes: Ulysses, J. Geophys. Res., 101, 11 027-11 038, 1996.

Tsurutani, B. T., Lakhina, G. S., Neubauer, F. M., and Glassmeier, K.-H.: A new look at the nature of comet Halley's LF electromagnetic waves: Giotto observations, Geophys. Res. Lett., 24, 3129-3132, 1997a.

Tsurutani, B. T., Glassmeier, K.-H., and Neubauer, F. M.: A review of nonlinear low frequency (LF) wave observations in space plasmas: On the development of plasma turbulence, in: Nonlinear Waves and Chaos in Space Plasmas, edited by: Hada, T. and Matsumoto, H., Terra Sci., Tokyo, 1, 1997b.

Tsurutani, B. T. and Lakhina, G. S.: Some basic concepts of waveparticle interactions in collisionless plasmas, Rev. Geophys., 35, 491-502, 1997.

Tsurutani, B. T., Lakhina, G. S., Winterhalter, D., Arballo, J. K., Galvan, C., and Sakurai, R.: Energetic particle cross-field diffu- sion: Interaction with Magnetic Decreases (MDs), Nonlin. Proc. Geophys., 6, 235-242, 1999,

\section{SRef-ID: 1607-7946/npg/1999-6-235.}

Tsurutani, B. T. and Ho, C. M.: A review of discontinuities and Alfvén waves in interplanetary space: Ulysses results, Rev. Geophys., 37, 517, 1999.

Tsurutani, B. T. and Lakhina, G. S.: Plasma microstructures in the solar wind, Proc Int. Sch. "Enrico Fermi" Course CXLII, edited by: Coppi, B., Ferrari, A., and Sindori, E., 257-272, 2000.

Tsurutani, B. T., Arballo, J. K., Galvan, C., Zhang, L., Zhou, X.Y., Lakhina, G. S., Hada, T., Pickett, J. S., and Gurnett, D. A.: Polar cap boundary layer waves: An auroral zone phenomenon, J. Geophys. Res., 106, 19 035-19056, 2001.

Tsurutani, B. T., Galvan, C., Arballo, J. K., Winterhalter, D., Sakurai, R., Smith, E. J., Buti, B., Lakhina, G. S., and Balogh, A.: Relationship between discontinuities, magnetic holes, magnetic decreases, and nonlinear Alfvén waves: Ulysses observations over the solar poles, Geophys. Res. Lett., 29, 23-1, doi:10.1029/2001GL013623, 2002a.

Tsurutani, B. T., Dasgupta, B., Galvan, C., Neugebauer, M., Lakhina, G. S., Arballo, J. K., Winterhalter, D., Goldstein, B. E., and Buti, B.: Phase-steepened Alfvén waves, proton perpendicular energization and the creation of magnetic holes and magnetic decreases: The ponderomotive force, Geophys. Res. Lett., 29, 86-1, doi:10:1029/2002GL015652, 2002b.

Tsurutani, B. T., Dasgupta, B., Arballo, J. K., Lakhina, G. S., and Pickett, J. S.: Magnetic field turbulence, electron heating, magnetic holes, proton cyclotron waves, and the onset of bipolar pulse (electron hole) events: A possible unifying scenario, Nonlin. Proc. Geophys., 10, 27-35, 2003,

\section{SRef-ID: 1607-7946/npg/2003-10-27}

Tu, C.-Y. and Marsch, E.: MHD structures, waves and turbulence in the solar wind-observations and theories, Spa. Sci. Rev., 73, 1-210, 1995.

Turner, J. M., Burlaga, L. F., Ness, N. F., and Lemaire, J. F.: Magnetic holes in the solar wind, J. Geophys. Res., 82, 1921-1924, 1977.

Vasquez, B. J. and Hollweg, J. V.: Formation of pressure balance structures and fast waves from nonlinear Alfvén waves, J. Geophys. Res., 104, 4681-4696, 1999.

Vasquez, B. J. and Hollweg, J. V.: Evolution and dissipation of imbedded rotational discontinuities and Alfvén waves in nonuniform plasma and the resultant proton heating, J. Geophys. Res., 106, 5661-5682, 2001.

Winterhalter, D., Neugebauer, M., Goldstein, B. E., Smith, E. J., Bame, S. J., and Balogh, A.: Ulysses field and plasma observations of magnetic holes in the solar wind and their relation to mirror-mode structures, J. Geophys. Res., 99, 23 371-23 382, 1994.

Winterhalter, D., Neugebauer, M., Goldstein, B. E., Smith, E. J., Tsurutani, B. T., Bame, S. J., and Balogh, A.: Magnetic holes in the solar wind and their relation to mirror-mode structures, Space Sci. Rev. 72, 201-204, 1995.

Winterhalter, D., Smith, E. J., Neugebauer, M., Goldstein, B. E., and Tsurutani, B. T.: The latitudinal distribution of solar wind magnetic holes, Geophys. Res. Lett., 27, 1615-1618, 2000.

Zhou, X.-Y. and Tsurutani, B. T.: Rapid intensification and propagation of the dayside aurora: large scale interplanetary pressure pulses (fast shocks), Geophys. Res. Lett., 26, 1097-1100, 1999. 